\title{
3 Research Square

\section{H3K4me3 and H3K27ac Activated IncRPL34-AS1 Acts as a Suppressor of Esophageal Squamous Cell Carcinoma via Targeting miR-575/ACAA2 Axis and Binding to ALOX12B and CAT}

Hu Zhang

Southeast University School of Public Health https://orcid.org/0000-0002-5358-6610

\section{Enchun Pan}

Huaian Center for Diease Control and Prevention

Ying Zhang

Southeast University School of Public Health

Chao Zhao

Southeast University School of Public Health

Qiwei Liu

Southeast University School of Public Health

Qiang Lu

Southeaast University School of Public Health

Jingjing Zhou

Southeast University School of Public Health

Ran Liu

Southeast University School of Public Health

Shizhi Wang

Southeast University School of Public Health

\section{Yuepu Pu}

Southeast University School of Public Health

Lihong Yin ( $\square$ lhyin@seu.edu.cn )

School of Public Health, Southeast University

\section{Research}

Keywords: ESCC, IncRPL34-AS1, miR-575, ACAA2, Histone modification, ALOX12B, CAT

Posted Date: November 12th, 2020

DOI: https://doi.org/10.21203/rs.3.rs-103657/v1 
License: (c) (i) This work is licensed under a Creative Commons Attribution 4.0 International License. Read Full License 


\section{Abstract}

Background: Long noncoding RNAs (IncRNAs) are abnormally expressed in a broad type of cancers and play significant roles that regulate tumor development and metastasis. However, the pathological roles of IncRNAs in esophageal squamous cell carcinoma (ESCC) remain largely unknown. Here we aimed to investigate the role and regulatory mechanism of the novel IncRPL34-AS1 in the development and progression of ESCC.

Methods: The expression level of IncRPL34-AS1 in ESCC tissues and different cell lines was determined by quantitative real-time PCR (RT-qPCR). Chromatin immunoprecipitation (ChIP) assay was used to evaluate the regulatory effect of histone modification on IncRPL34-AS1. Then, functional experiments in vitro and in vivo were employed to explore the effects of IncRPL34-AS1 on tumor growth and metastasis in ESCC. Mechanistically, fluorescence in situ hybridization (FISH), bioinformatics analyses, luciferase reporter assay, RNA immunoprecipitation (RIP) assay and western blot assays were used to detect the regulatory relationship between IncRPL34-AS1, miR-575 and ACAA2. In addition, comprehensive identification of RNA binding proteins (ChIRP), mass spectrometry, and RIP assay were used to identify IncRPL34-AS1-interacting proteins.

Results: LncRPL34-AS1 was significantly down-regulated in ESCC tissues and cells, which was negatively correlated with overall survival in ESCC patients. The chromatin immunoprecipitation (ChIP) assays indicated that gain of H3K4me3 and H3K27 acetylation-activated IncRPL34-AS1 was down-regulated in ESCC. Functionally, upregulation of IncRPL34-AS1 dramatically suppressed ESCC cell proliferation, colony formation, cell cycle progression and induced apoptosis in vitro, whereas knockdown of IncRPL34AS1 elicited the opposite function. Consistently, overexpression of IncRPL34-AS1 inhibited tumor growth and metastasis in vivo. Mechanistically, IncRPL34-AS1 acted as competing endogenous RNA (ceRNA) of miR-575 to relieve the repressive effect of miR-575 on its target ACAA2, then suppressed the tumorigenesis of ESCC. In addition, protein ALOX12B and CAT resulted direct binding targets of IncRPL34-AS1 and affected biological process in ESCC.

Conclusions: Together, our results reveal a role for IncRPL34-AS1 in ESCC tumorigenesis and may provide a strategy for using IncRPL34-AS1 as a potential biomarker and a therapeutic target for patients with ESCC.

\section{Background}

Esophageal carcinoma (EC), as one of current malignant digestive system cancers, causes the sixth mortality rate cancer in the worldwide and the fourth incident rate in China [1, 2]. According to the latest Global Cancer Statistics 2018, the number of EC cases were as high as 572034 [1]. The regions with the highest incidence of EC in recent years have been concentrated in areas stretching from East Asia to Central Asia [3]. Esophageal squamous cell carcinoma (ESCC), the major type of EC in China accounting for approximately $90 \%$ of EC cases, remains one of the most lethal of malignancies and a major health 
burden [4]. The prognosis of EC remains enormously poor, with a low overall five-year survival rate of about $10 \%[5,6]$. Therefore, it is necessary to provide novel insights into the underlying mechanisms of ESCC progression, which can identify potential therapeutic targets to improve patient survival.

Studies have revealed that $98 \%$ of human genome transcripts RNAs are non-coding RNA (ncRNAs) with limited or no protein coding capabilities. LncRNAs, larger than 200 nucleotides, participate in almost all human biological processes, including transcriptional regulation, epigenetic regulation, cell differentiation, embryonic development, and key signaling of cancer as well as other diseases $[7,8]$. Some studies show that IncRNAs are involved in numerous important regulatory processes such as $\mathrm{X}$ chromosome silencing, genomic imprinting and chromatin modification, transcriptional activation, transcriptional interference, and nuclear transport [9]. Abnormal expression of IncRNAs in various types of cancer illustrates clinical potential as biomarkers and therapeutic targets [10]. At present, studies on the occurrence and development of IncRNA mediated regulation on ESCC progression are in full swing [11]. LncRNA acts as competing endogenous RNAs (ceRNAs) through harboring miRNA, thus modulating miRNA target gene expression, which promotes metastasis and development of ESCC [12, 13]. Also IncRNA could interact with RNA-binding proteins and regulate signaling pathways to impose transcriptional regulation, which can serve as potential prognostic biomarkers and therapeutic targets for ESCC $[14,15]$.

As a novel discovered IncRNA, IncRPL34-AS1 has attracted increasing attention and research [16]. Overexpression of IncRPL34-AS1 inhibits colorectal cancer cell proliferation, invasion, and apoptosis, in which may play a regulatory role through the IncRPL34-AS1/miR-93/PTEN axis [17]. Validation of paired gastric cancer specimens found that decreased expression of IncRPL34-AS1 was normally correlated with larger cancer tissue sizes [18]. LncRPL34-AS1 acts a suppressor of thyroid papillary carcinoma via competitively binding miR-3663-3p/RGS4 axis [19].

Our preliminary research identified IncRPL34-AS1 which was significantly associated with the overall survival of the esophageal cancer patients [20]. In this study, we verified that IncRPL34-AS1 was downregulated in ESCC tissues and cell lines. A unique role for IncRPL34-AS1 in suppressing ESCC growth and metastasis was demonstrated by gain- and loss-of-function experiments in vitro and in vivo. H3K4me3 and H3K27 acetylation enrichment activated downregulation on the promoter near the transcription start site of IncRPL34-AS1. In addition, IncRPL34-AS1 regulated miR-575/ACAA2 axis to suppress ESCC growth and metastasis. We demonstrated that InCRPL34-AS1 specifically bound to protein ALOX12B and CAT which affected biological processes of ESCC. In brief, our results provide the function and mechanism of IncRPL34-AS1 in ESCC progression, which may assist in the development of new therapeutic targets for ESCC intervention.

\section{Material And Methods}

\section{Tissue samples and ethics statement}


A total of 75 primary pairs of ESCC tissue samples and adjacent normal tissues were obtained from Huai'an First People's Hospital of Jiangsu Province, China. None of ESCC patients received any radiotherapy or chemotherapy treatments before surgery. After separated, the clinical specimens were immediately put into liquid nitrogen and transferred to $-80^{\circ} \mathrm{C}$ for preservation. This study was approved by the Southeast University Affiliated Zhongda Hospital Ethics Committee, and it was conducted in compliance with the Helsinki Declaration. All eligible participants have signed written informed consent for publication.

\section{Cell culture}

The normal human esophageal squamous epithelial cell line (Het-1A), and human ESCC cell lines (EC109, EC9706) were provided by the Key Laboratory of the Environmental Medicine Engineering of Southeast University, Ministry of Education, China. EC109 and EC9706 cells were cultured in RPMI-1640 medium (Gibco, USA) and Het-1A was maintained in DMEM medium (Gibco, USA), respectively. The cell culture medium supplemented with $10 \%$ fetal bovine serum (Biological Industries), $1 \%$ penicillin and streptomycin (Gibco, USA) in humidified air at $37^{\circ} \mathrm{C}$ with $5 \% \mathrm{CO}_{2}$.

\section{RNA extraction and real-time quantitative polymerase chain reaction (RT-qPCR)}

The total RNA extraction, reverse transcription and real-time quantitative PCR of IncRPL34-AS1, miRNA and mRNA expression were performed as previously described [21]. The detailed sequences of primers were listed in Additional file 1: Table 1. 
Table 1

Correlation between the clinicopathologic characteristics and RPL34-AS1 expression in ESCC

\begin{tabular}{|c|c|c|c|c|c|}
\hline \multirow{3}{*}{$\begin{array}{l}\text { Characteristics } \\
\text { Age (y) }\end{array}$} & \multirow{3}{*}{$\begin{array}{l}\text { Class } \\
<50\end{array}$} & \multirow{3}{*}{$\begin{array}{l}n=75 \\
3\end{array}$} & \multicolumn{2}{|c|}{ RPL34-AS1 } & \multirow[t]{2}{*}{$P$} \\
\hline & & & \multicolumn{2}{|c|}{ Low expression High expression } & \\
\hline & & & 0 & 3 & \multirow[t]{2}{*}{$0.008^{\star}$} \\
\hline & $\geq 50$ & 72 & 59 & 13 & \\
\hline \multirow[t]{2}{*}{ Sex } & Male & 53 & 40 & 13 & \\
\hline & Female & 22 & 19 & 3 & 0.367 \\
\hline \multirow[t]{3}{*}{ Tumor site } & lower & 17 & 11 & 6 & \multirow[t]{3}{*}{0.671} \\
\hline & middle & 50 & 43 & 7 & \\
\hline & upper & 7 & 4 & 3 & \\
\hline \multirow[t]{2}{*}{ Lymph node metastasis } & no & 52 & 43 & 9 & \multirow[t]{2}{*}{0.078} \\
\hline & yes & 23 & 16 & 7 & \\
\hline \multirow[t]{3}{*}{ Smoking } & light & 28 & 24 & 4 & \multirow[t]{3}{*}{0.158} \\
\hline & middle & 9 & 5 & 4 & \\
\hline & deep & 38 & 30 & 8 & \\
\hline \multirow[t]{2}{*}{ Drinking } & no & 44 & 36 & 8 & \multirow[t]{2}{*}{0.568} \\
\hline & yes & 31 & 23 & 8 & \\
\hline \multirow[t]{2}{*}{ Hot food } & no & 38 & 30 & 8 & \multirow[t]{2}{*}{1.000} \\
\hline & yes & 37 & 29 & 8 & \\
\hline \multirow[t]{2}{*}{ Hard food } & no & 46 & 40 & 6 & \multirow[t]{2}{*}{$0.042^{\star}$} \\
\hline & yes & 29 & 19 & 10 & \\
\hline \multirow[t]{2}{*}{ Fried food } & no & 53 & 39 & 14 & \multirow[t]{2}{*}{0.496} \\
\hline & yes & 13 & 11 & 2 & \\
\hline \multirow[t]{2}{*}{ History of digestive disease } & no & 65 & 50 & 15 & \multirow[t]{2}{*}{0.679} \\
\hline & yes & 10 & 9 & 1 & \\
\hline
\end{tabular}

\section{Cell transfection}

EC109 cells were transfected with siRNAs and plasmid vectors using StarFect $\$ High-Efficiency Transfection Reagent (GenStar) according to the manufacturer's protocol. Three individual IncRPL34-AS1 
siRNAs (si-RPL34-AS1 1\#, 2\# and 3\#) and plasmid vector (pcDNA-IncRPL34-AS1, pcDNA3.1 empty vectors) were purchased from KeyGEN BioTECH. The miR-575 mimics, miR-575 inhibitor and pcDNAACAA2 were provided by Genomeditech. The all nucleotide sequences were listed in Additional file 1: Table 2. After 48 hours post-transfection, cells were harvested for RT-qPCR or Western blot analysis.

\section{Cell proliferation, invasion and migration assays}

The in vitro invasion, migration, CCK8, colony formation and EdU proliferation assay were detected as previous described [21, 22].

\section{Western blot assay}

Western blot assay was performed as previous described [22]. Antibodies: ACAA2 (1:1000, Abcam), $\beta$ actin (1:2000, Cell Signaling Technology), Cyclin D1 (1:1000, Abcam), Bcl-2 (1:1000, Abcam) and antiBAX (1:2000, Abcam). The signals were detected by the SuperSignal West Femto Trial Kit (Thermo Fisher Scientific).

\section{Flow cytometric assay}

Cell apoptosis was quantified using the Annexin V-FITC Apoptosis Detection Kit (KGA107) and the Annexin V-APC/7-AAD (KGA1024, Keygen Biotech, Nanjing, China) in accordance with the manufacture's protocol. The cell cycle was performed using PI cell cycle Detection Kit (KGA107, Keygen Biotech) according to the manufacture's protocol.

\section{Dual-luciferase reporter assay}

The sequences of IncRPL34-AS1 and ACAA2-3'UTR and their corresponding mutations were designed, synthesized and inserted into luciferase reporter vector PGL3-CMV-LUC-MCS (Genomeditech, Shanghai). The relative ratio of firefly luciferase (Luc) activity/Renilla luciferase (Rena) activity was applied to evaluate fluorescence intensity.

\section{RNA immunoprecipitation (RIP)}

The RNA immunoprecipitation (RIP) assay was performed using EZ-Magna RIP Kit (EMD Millipore, Billerica, MA) following the manufacturer's protocol. The lysed cell was immunoprecipitated with antiArgonaute 2 (AGO2), anti-CAT, anti-ALOX12B and anti-IgG antibody (EMD Millipore). Finally, the purified RNA was detected by RT-qPCR analysis.

\section{RNA fluorescent in situ hybridization (FISH)}

To localize the cellular distribution of IncRPL34-AS1, the FISH assay was performed using the IncRNA FISH Probe and Fluorescent in Situ Hybridization Kit (Ribobio, China) according to the manufacturer's guidelines. The probe cocktail included the $18 \mathrm{~S} /$ cytoplasm probe, U6/nuclear probe, and IncRPL34-AS1 probe (DAPI and Cy3-labeled probes were synthesized for fluorescence signals). The results were captured by the OLYMPUS laser confocal microscope FV1000 (Olympus, Tokyo, Japan).

\section{Chromatin immunoprecipitation (ChIP) assay}


The ChIP assays were performed using ChIP Assay Kit (Cell Signaling Technology). EC109 and Het-1A cells with $37 \%$ formaldehyde were incubated for 10 minutes at room temperature to form DNA-protein crosslinks, and then cross-linked chromatin DNAs were broken into 200 to 1000 bp-sized segments by using an ultrasound breaker. Anti-H3K27ac antibody, anti-H3K4Me3 antibody or IgG antibody (Cell Signaling Technology) was used to precipitate the chromatin the lysate. After de-crosslinked enriched target of protein-DNA complex, and finally the enriched DNA fragments were purified and analyzed via RTqPCR.

\section{Detection of MDA and SOD levels}

The malondialdehyde (MDA) content and superoxide dismutase (SOD) activity were examined by commercial detection kits (Beyotime, Shanghai, China) following the manufacturer's instructions.

\section{In vivo transcription assays and ChIRP-Mass spectrometry (LC-MS/MS) assays}

12-20 $15 \mathrm{~cm}$ dishes of cells were used per ChIRP-MS experiment (200 million EC109 cells). The IncRPL34-AS1 in vivo probe labeled with biotin was provided by Akimics. Cell harvesting, lysis, disruption, pre-bind probe to streptavidin beads for ChIRP. After tryptic digestion and peptide desalting, for each sample, peptides were separated and analyzed with a nano-UPLC (EASY-nLC1200) coupled to Q-Exactive mass spectrometry (Thermo Finnigan). The raw MS files were processed with MaxQuant (Version 1.5.6.0) database search and label free quantitative analysis. Subsequently, the quantitative results of the samples were statistically analyzed, and the corresponding enriched proteins were obtained based on the FCa value and number of unique peptides. Bioinformatics analysis such as GO, KEGG pathway, and protein interaction of comprehensive RNA-Binding proteins-Mass Spectrometry results were analyzed and displayed.

\section{Transcriptome sequencing (RNA-seq) and computational analysis}

EC109 cells transfected by IncRPL34-AS1 plasmid and scramble pcDNA were used for RNA-sEq. RNA quantification and quality assurance were evaluated by NanoDrop ND-1000, and RNA integrity and gDNA contamination tested by standard denaturing agarose gel electrophoresis. The mixed different sample libraries were sequenced by IlluminaNovaSeq6000 sequencer; raw sequencing data performed sequencing quality control (QC) to evaluate sequencing data analysis. The R software Ballgown was used to calculate the FPKM at gene level and transcript level, and the differences of expression at gene level and transcript level were calculated respectively. Based on the PCA analysis and correlation analysis of gene and transcript expression level, the differentially expressed genes were analyzed by scatter plot, volcano diagram, cluster diagram, GO function and KEGG pathway significant enrichment. Accession ID for the RNA-seq data is GSE154450.

\section{Tumor xenograft model}


To study the effect of IncRPL34-AS1 on tumor growth and metastasis, BALB/c nude mice (male, 4-weekold) were obtained for tumor xenografts experiments (Jiangsu GemPharmatech, China). In tumor growth assay in vivo, EC109 cells transfected with IncRPL34-AS1-overexpressing or control vector were subcutaneously injected into the right flank of the mice $\left(5 \times 10^{6}, 200 \mu \mathrm{l}\right)$. Volumes of tumors were measured every 3 days after being apparently observed and calculated with the following formula: Volume $=\left(\right.$ length $\times$ width $\left.^{2}\right) / 2$. After 2 weeks of tumor generated, mice were sacrificed and subcutaneous tumor tissues were detected for tumor weight, RT-qPCR and Hematoxylin and eosin (H\&E) staining. The antibodies against Ki-67 (Abcam, Cambridge, MA, USA) were applied for immunohistochemistry (IHC). In tumor metastasis in vivo, IncRPL34-AS1-overexpressing or control EC109 cells $\left(1 \times 10^{6}, 200 \mu \mathrm{l}\right)$ were intravenously injected into tail vein of nude mice. After 34 days, the livers and lungs were removed, paraffin-embedded and finally validated by H\&E staining. All animal procedures were approved by the Animal Care and Use Committee of Southeast University.

\section{Statistical analysis}

Data were analyzed using SPSS 23.0 (IBM, USA) and GraphPad Prism8.1 software. All results were expressed as the mean \pm SD. The Student's t-test and one-way ANOVA were performed to analyze whether two or more groups had statistical significance. Chi-square test was used to estimate the correlation between the IncRPL34-AS1 expression and clinicopathologic features. Overall survival curves were protracted using the Kaplan-Meier method and estimated by the log-rank test. The gene expression correlation was analyzed using the Pearson correlation test. A two-sided $P$ value $<0.05$ was considered as statistically significant.

\section{Results}

\section{Gain of H3K4me3 and H3K27 acetylation-activated IncRPL34-AS1 is down-regulated in ESCC and negatively correlated with poor prognosis}

To explore the role of RPL34-AS1 in ESCC, we first assessed RPL34-AS1 expression level in 75 paired primary ESCC and matched adjacent nontumor tissues by RT-qPCR. The results showed that the expression of RPL34-AS1 was significantly reduced in the ESCC tissue samples (Fig. 1a). Moreover, RPL34-AS1 expression was down-regulated in ESCC cell lines compared with Het-1A cells, Among ESCC cell lines, EC109 cells showed the significant downregulation. Thus, we selected EC109 cell line to investigate the downstream regulatory pathway of RPL34-AS1 (Fig. 1b). Furthermore, the results of FISH assay showed that the RPL34-AS1 transcripts were distributed both in the cytoplasm and nucleus of EC109 cells (Fig. 1c), which indicated RPL34-AS1 might function in both cytoplasm and nucleus. To further investigate the expression pattern of RPL34-AS1 in ESCC, we also performed an analysis of RPL34-AS1 expression in a public microarray profile dataset from the Cancer Genome Atlas (TCGA). Consistent with our previous results, RPL34-AS1 expression was downregulated in ESCC tissues (Fig. 1d). Then we divided all ESCC patients into high and low RPL34-AS1 expression level groups according to the median value. Kaplan-Meier survival analysis of ESCC patients with low RPL34-AS1 expression had a 
significant poorer overall survival than those with high RPL34-AS1 expression ( $P=0.01788$, Fig. 1e). As shown in Table 1, the correlation analysis between RPL34-AS1 expression and clinicopathologic characteristics of these ESCC patients indicated that low expression of RPL34-AS1 was positively correlated with age $(P=0.008)$ and hard food $(P=0.042)$.

To explore the epigenetic modification mechanism of IncRPL34-AS1 in ESCC, firstly, using UCSC Genome Bioinformatics Site (http://genome.ucsc.edu/), we found high enrichment of H3K4me3 and H3K27ac at the promoter of RPL34-AS1. Hence, we speculated that the downregulation of RPL34-AS1 could be attributed to $\mathrm{H} 3 \mathrm{~K} 4 \mathrm{me} 3$ and $\mathrm{H} 3 \mathrm{~K} 27 \mathrm{ac}$ at its promoter region. To confirm this hypothesis, we detected the gain of H3K4me3 and H3K27Ac in EC109 cells compared with Het-1A cells at the promoter of RPL34-AS1 (Fig. 1f). Together, the data above confirmed that RPL34-AS1 was frequently reduced in ESCC, histone methylation and acetylation activation of promoter may partly account for this dysregulation.

\section{LncRPL34-AS1 suppresses ESCC cell proliferation, cell- cycle progression, migration, invasion and promotes cell apoptosis in vitro}

Given that RPL34-AS1 was down-regulated in ESCC, loss- and gain-of-function approaches were employed to determine the biological function of RPL34-AS1 in ESCC cells. RPL34-AS1-overexpressing EC109 cell line was established by the transfection of pcDNA3.1-RPL34-AS1. In contrast, three siRNAs were designed to silence RPL34-AS1. Real-time PCR analysis confirmed that RPL34-AS1 expression was successfully down-regulated or up-regulated in EC109 cells (Fig. 2a). CCK-8 assays demonstrated that downregulation of RPL34-AS1 significantly enhanced the proliferation viability, whereas the upregulation of RPL34-AS1 exerted opposite effects (Fig. 2b). Colony formation assays further demonstrated that the cell cloning capabilities of EC109 were obviously enhanced by the downregulation of RPL34-AS1 and markedly impaired by the overexpression of RPL34-AS1 (Fig. 2c). Similarly, EdU assays revealed that knockdown of IncRP34-AS1 greatly increased the percentages of EdU-positive cells, which considerably decreased at overexpression of RPL34-AS1 (Fig. 2d). Moreover, flow cytometry assays revealed that the percentage of apoptotic ESCC cells was reduced by RPL34-AS1 knockdown and the overexpression of RPL34-AS1 promoted the ESCC cells apoptosis (Fig. 2e). Depletion of RPL34-AS1 promoted cell cycle progression and upregulation of RPL34-AS1 induced cell cycle arrest at G1/S phase in EC109 cells (Fig. 2f). Furthermore, western blot results showed the consistent trend of cell apoptosis and cycle that RPL34-AS1 knockdown led to increase the levels of Bcl-2, Cyclin D1 and decrease BAX expression, as well as the upregulation of RPL34-AS1 led to opposite effects (Fig. 2g).

Next, transwell assays were carried out to examine the effects of RPL34-AS1 on migration and invasion of EC109 cells. The results indicated that the migratory and invasive capabilities of EC109 cells were remarkably enhanced by downregulation of RPL34-AS1 but significantly suppressed by upregulation of RPL34-AS1 (Fig. 2h, i). These experiments suggested that IncRPL34-AS1 suppressed migration and invasion of EC109 cells. 


\section{Overexpression of IncRPL34-AS1 restrains growth and metastasis of ESCC in vivo}

To further determine the effects of IncRPL34-AS1 on tumor growth in vivo, EC109 cells transfected with pc-RPL34-AS1 or control vector were subcutaneously injected into BALB/c nude mice. 34 days after injection, the tumors were collected. The tumors formed in the pc-RPL34-AS1 group were substantially smaller than those in the control group (Fig. 3a). The results of tumor growth curves and weights indicated that RPL34-AS1 overexpression obviously reduced tumor growth in mice (Fig. 3b, c). Tumor tissues were harvested for RT-qPCR analysis of RPL34-AS1 and ACAA2. We confirmed that higher expression of RPL34-AS1 and ACAA2 were detected in tumor tissues arising from RPL34-AS1 overexpression group compared to control group (Fig. 3d, e). Furthermore, H\&E and IHC for Ki67 were performed to detect the expression of Ki67, and results showed that RPL34-AS1 overexpression caused decreased Ki67 expression (Fig. 3f). To investigate the role of IncRPL34-AS1 in tumor metastasis, EC109 cells transfected with pcRPL34-AS1 or control vector were injected into the tail vein of nude mice. Compared with the control group, the IncRPL34-AS1 over-expression group blunted lower lung metastasis (Fig. 3g). Altogether, these results suggested that RPL34-AS1 upregulation suppressed ESCC tumorigenesis in vivo.

\section{LncRPL34-AS1 serves as a miRNA sponge of miR-575 to regulate ACAA2 expression}

To investigate the mechanisms underlying the role of RPL34-AS1 in ESCC, we examined the mRNA expression profiles in EC109 cells after overexpression of IncRPL34-AS1. The cluster analysis, volcano plot, GO and KEGG enrichment of differentially expressed genes show in Additional file 2: Figure. S1. The miRNA information in miRBase was used to perform target prediction based on the RPL34-AS1 sequence and the candidate miRNAs were screened by miRanda and TargetScan algorithm to display the ternary relationship of IncRNA-miRNA-mRNA in the form of a network diagram (Fig. 4a). We used differentially expressed genes of RNA-seq results and competing endogenous RNAs (ceRNA) targets to take the intersection in Venny (Fig. 4b). After DAVID bioinformatics resources functional notes, three metabolic pathways were enriched (Fig. 4c) and 6 targeted genes were selected for subsequent mechanism research in line with fold change and $p$ value. The results of ACAA2 gene expression were proved to show consistent trend with RPL34-AS1 after knockdown and overexpression of RPL34-AS1 in EC109 cells (Fig. 4d, e). Correlated with previous ceRNAs prediction, the candidate miR-575 was selected for targeting ACAA2 and IncRPL34-AS1 via miRbase predicting (Fig. 4f). In brief, the IncRPL34-AS1/miR-575/ACAA2 regulatory network was established for verifying in ESCC.

Next, we detected the expression of miR-575 and ACAA2 in ESCC and matched adjacent normal tissues. Results of RT-qPCR showed significant upregulation of miR-575 and downregulation of ACAA2 in ESCC tissues relative to adjacent normal tissues (Fig. 4g). Furthermore, the results of RT-qPCR in EC109 cells also showed significant upregulation of miR-575 relative to Het-1A cells (Fig. 4h). Correspondingly, we then exemplified the binding relationship between IncRPL34-AS1 and miR-575; we conducted a wild-type 
(WT) and mutant (MUT) PGL3-CMV-IncRPL34-AS1 vector containing the binding sites of miR-575. The results showed that the luciferase activity of WT IncRPL34-AS1 reporter vector was significantly reduced by miR-575 mimics, compared with the empty vector and mutant reporter vector (Fig. 4i). Followed by an anti-AG02 RIP assay was implemented to validate the binding relationship between IncRPL34-AS1 and miR-575, the results of RIP implied that the expression of miR-575 in the IncRPL34-AS1 overexpression group was specifically higher than the NC group (Fig. 4j).

To decipher the regulatory mechanisms of miR-575 on ACAA2, we transfected luciferase reporter vector harboring 3' UTR (WT and MuT) of ACAA2 into EC109 cells and luciferase activity was then evaluated in the transfection of miR-575 mimics. As compared to the control vector, miR-575 mimics significantly reduced the luciferase activity of the ACAA2 reporter vector (ACAA2 3' UTR-WT) (Fig. 4k). Furthermore, to confirm the role of IncRPL34-AS1 on regulation of miR-575/ACAA2, we then set up another dualluciferase (DLR) analysis and divided into two groups: Group 1 (RPL34-AS1 + ACAA2 WT + miR-575 mimics) and Group 2 (NC + ACAA2 WT + miR-575 mimics), the fluorescence intensity in Group 2 was reduced by $31 \%$ compared with Group 1 (Fig. 4I). These results enlightened that IncRPL34-AS1 may regulate ACAA2 expression by competitively interacting with miR-575.

\section{LncRPL34-AS1 suppresses ESCC cell growth and metastasis through IncRPL34-AS1/miR-575/ACAA2 axis}

Accordingly, to verify whether RPL34-AS1 served its tumor suppressor function through RPL34-AS1/miR575/ACAA2 axis, rescue experiments were designed using inhibitors and mimics. As shown in Fig. 5a, knockdown of RPL34-AS1 decreased protein level of ACAA2 in EC109 cells, while upregulation of RPL34AS1 enhanced the level of ACAA2 in EC109 cells. As few studies had explored the role of miR-575 in ESCC, we began to clarify the mechanism and biological function of miR-575 in EC109 cells. The results indicated that upregulation of miR-575 significantly enhanced the proliferation viability, migratory and invasive capabilities of EC109 cells (Fig. 5b, c).Simultaneously, the target mRNA ACAA2 expression caused by silencing or overexpressing RPL34-AS1 were reversed by miR-575 inhibitor or mimics, respectively (Fig. 5d). Moreover, we attempted to explore whether the biological function of RPL34-AS1 in EC109 cells could also be reversed by miR- 575 inhibitor or mimics. The results indicated that the miR- 575 mimics reversed the proliferation, migration and invasion inhibiting effects induced by overexpression of RPL34-AS1 in EC109 cells, whereas miR-575 inhibitor counteracted the promoting effects induced by knockdown of RPL34-AS1 in EC109 cells (Fig. 5e-h).

Also we performed the mechanistical function of miR-575 by targeting ACAA2. The results of RT-qPCR and western blot displayed that substantially increased expression of ACAA2 in miR-575 mimics group in EC109 cells (Fig. 5i). Furthermore, the overexpression of ACAA2 (pcACAA2) repressed the proliferation viability, migratory and invasive capabilities of EC109 cells, whereas the above effects were reversed by miR-575 mimics (Fig. 5j-m). In addition, the flow cytometry assays also revealed that the cell cycle progression was promoted after co-transfected by pcACAA2 and miR- 575 mimics, as well as the same trend that co-transfected by RPL34-AS1 upregulation and miR-575 mimics (Fig. 5n). In summary, these 
data strongly suggest that IncRPL34-AS1 suppresses ESCC cell growth and metastasis through IncRPL34-AS1/miR-575/ACAA2 axis.

\section{LncRPL34-AS1 affects biological processes via binding to protein ALOX12B and CAT}

To search for the potential interacting molecules of RPL34-AS1 to regulate target genes at distal genomic loci, we purified endogenous RPL34-AS1 complexes using modified ChIRP [23] that allowed unbiased high-throughput discovery of RPL34-AS1 associated binding proteins, ChIRP-MS was optimized to identify IncRNA-associated proteins (Fig. 6a). We designed 38 probes (even/odd group) against human RPL34-AS1 RNA (Additional file 1: Table 3). The total TIC peaks of Positive Control (U1 snRNA), Negative control (Ctrl) and Test sample (Lnc) resolved components displayed in Fig. 6b. Protein enrichment classification information for comparison Lnc-Ctrl were displayed as Venn diagram, and the results showed a number of 73 proteins that can bind to RPL34-AS1 (Fig. 6c). The results of STRINGdb proteinprotein network enrichment analysis indicated the correlation between differential proteins (Fig. 6d). The KEGG pathways were enriched that Ribosome and Glycolysis/ Gluconeogenesis exerted significantly effect on interaction between IncRPL34-AS1 and binding proteins (Fig. 6e). According to the iBAQ and fold change, we selected 23 functional proteins for subsequent study (Fig. 6f). As shown in Fig. 6g, the functional protein pathway Glycolysis/ Gluconeogenesis was related to IncRPL34-AS1 in ESCC.

Among all the ChIRP-retrieved proteins, catalase (CAT) and Arachidonate 12-lipoxygenase, 12R-type (ALOX12B) came into notice. The protein ALOX12B catalyzes the region and stereo-specific incorporation of a single molecule of dioxygen into free and esterified polyunsaturated fatty acids generating lipid hydroperoxides [24]. The catalase occurs in almost all aerobically respiring organisms and serves to protect cells from the toxic effects of hydrogen peroxide [25]. The results of MDA and SOD detection confirmed the correlation between IncRPL34-AS1 and cellular oxidative stress (Fig. 6h). To further confirm the relationship, we examined the interaction between RPL34-AS1 and the two proteins by RNA immunoprecipitation (RIP), the results showed significant enrichments of RPL34-AS1 bound to CAT and ALOX12B, compared with the non-specific IgG control (Fig. 6i). Also, western blot showed that CAT and ALOX12B protein were positively proportional to RPL34-AS1 at a posttranscriptional level (Fig. 6j). As previous results shown, the IncRPL34-AS1 regulated target gene ACAA2 was a part of lipid metabolism as well as ALOX12B. In addition, correlation was found between ALOX12B and ACAA2 mRNA in ESCC of TCGA database (Fig. 6k). Altogether, these data showed that RPL34-AS1 affected biological processes via protein ALOX12B and CAT in ESCC.

\section{Discussion}

LncRNA is encoded by a less explored region of the human genome and may retain impairing cancer drivers. Recently, it has attracted attention as a potential key layer of cancer cell regulation [26]. LncRNA determines its molecular function: cytoplasmic IncRNA plays an important functional role in regulating the translation and decay of messenger RNA, relying on competitive endogenous RNAs (ceRNAs) to 
sequester miRNAs from binding to their cognate mRNA targets and protein modification; nuclear IncRNA has functions at the transcriptional level such as histone modification, alternative splicing, or direct transcriptional regulation [27, 28]. Meanwhile, increasing studies have revealed that IncRNAs exerted biological and clinical relevance of metastasis-associated function in ESCC [29]. In the present study, we confirmed that the novel IncRPL34-AS1 was obviously downregulated in ESCC and significantly correlated with age and hard food as well as overall survival of ESCC patients. TCGA data also revealed that low expression of RPL34-AS1 indicated poor survival of patients with ESCC. Next, loss- and gain-offunction assays indicated that IncRPL34-AS1 acted as a tumor suppressor in vivo and vitro of ESCC. Given the cellular distribution of IncRPL34-AS1 in both nucleus and cytoplasm, we utilized histone modification, ceRNA regulatory network and RNA-binding proteins to explore the underlying molecular mechanisms of IncRPL34-AS1.

One of the main mechanisms of IncRNAs is their ability to function by binding to histone modification complexes and relaxing the structure of chromatins and improving transcriptional activity [30]. LncRNA can recruit chromatin modifiers to regulate the chromatin state, or directly regulate the transcription process through the chromosome loop, thereby bridging the distal enhancer to the promoter [31]. For instance, the gain of H3K4me3 and H3K27ac activation of the promoter facilitate activation of HOXCAS3 in gastric cancer [32]. Herein, we found through UCSC genome browser that H3K4me3 and H3K27ac were highly enriched at the promoter region of RPL34-AS1. Our results revealed that H3K4me3 and H3K27ac activation of promoter partly contributed to downregulation of RPL34-AS1 in ESCC through ChIP assays. Recent studies also demonstrated that H3K4me3 and H3K27ac were related to gene activation, which mainly enriched in the promoter region near the transcription start site [33, 34].

It is emerging that one of the most popular functional models by which IncRNAs regulate gene expression is to interact with miRNA as ceRNAs that bind to miRNA response elements (MREs) and repress targeting mRNAs $[35,36]$. Here, analyzed by bioinformatics analysis, IncRPL34-AS1 was predicted to harbor miRNA-binding sites of miR-575 in the IncRPL34-AS1 sequence, which was further confirmed by luciferase reporter assay and RIP assay. Furthermore, the expression of IncRPL34-AS1 was negatively associated with miR-575, and a significant reciprocal repression feedback loop present in ESCC cells. Importantly, miR-575 acted as a tumor promoter in ESCC, and subsequent rescue experiments further confirmed that miR-575 mimics or inhibitor reversed the tumor suppressor roles of IncRPL34-AS1. Together, our results revealed that IncRPL34-AS1 could serve as a ceRNA by sponging miR- 575 in ESCC.

Acetyl CoA acyltransferase 2 (ACAA2) is a key enzyme in the fatty acid oxidation pathway which catalyzes the last step of mitochondrial beta-oxidation and participates in various pathways related to lipid metabolism [37]. To date, whether and how IncRNA contributed to ACAA2-induced progression in ESCC remains elusive. In the present study, our results indicated that IncRPL34-AS1 interacted with miR575 to promote the expression of ACAA2, and inferred a novel mechanistic role of IncRPL34-AS1/miR575/ACAA2 axis in regulating the progression of ESCC. Furthermore, we revealed that ACAA2 was downregulated in ESCC and ACAA2 overexpression clearly inhibited the proliferation, migration and 
invasion of ESCC cells. Hence, we further demonstrated the oncogenic role of ACAA2 and provided evidence for the posttranscriptional regulation of ACAA2 by IncRPL34-AS1 in ESCC.

Up to now, accumulating evidence has illustrated that IncRNAs participate in enabling scaffolding functions and combinatorial control by binding with specific proteins [32]. Regulation roles of IncRNAs in the RNA-binding proteins interaction involve various aspects, such as altering protein localization, modulating protein expression and activity or serving as structural components [35, 38]. In our ChIRP and MS assays, IncRPL34-AS1 could interact with ALOX12B and CAT proteins, which was specifically verified by RIP assays. The correlation between IncRPL34-AS1 and CAT proved to be involved in cellular oxidative stress. Also, the target gene ACAA2 and binding protein ALOX12B of IncRPL34-AS1 all participate in lipid metabolism. Recent observations indicate that certain IncRNAs can bind directly or indirectly to lipids and play an important role in regulating lipid metabolism, lipid signaling and mitochondrial function [39-41]. Moreover, we found that the correlation between ACAA2 and ALOX12B was significantly statistic in TCGA database. As a result, IncRPL34-AS1 can be involved in the regulation of multiple lipid metabolism-related protein and oxidative stress protein in ESCC.

To our knowledge, this study is giving us a novel point of view for specific molecular targets in ESCC, and it deepened our understanding of the relationship between IncRNA, histone modification, miRNA and proteins in disease progression, whereas IncRPL34-AS1 regulated the development of ESCC through other mechanisms such as post-transcriptional regulation and signaling pathway feedback required further investigation. Therefore, a deeper understanding of the therapeutic potential of IncRPL34-AS1 in ESCC which warrants additional detailed studies.

\section{Conclusions}

In summary, we identified abnormal histone modification-mediated activation of a novel IncRNA RPL34AS1, which was obviously downregulated in ESCC and functioned as a tumor suppressor. Furthermore, we also demonstrated that IncRPL34-AS1 acted as an endogenous sponge of miR-575 and subsequently promoted ACAA2 expression to inhibit ESCC cell growth and metastasis. LncRPL34-AS1 may affect the ESCC progression via interacting with proteins, thus promoting transcription and translation process. As shown in Fig. 6l, our results reveal a role for IncRPL34-AS1 in ESCC tumorigenesis and may provide a strategy for using IncRPL34-AS1 as a potential biomarker and a therapeutic target for patients with ESCC.

\section{Abbreviations}




\begin{tabular}{|ll|}
\hline Abbreviation & Meaning \\
\hline ESCC & esophageal squamous cell carcinoma \\
\hline EC & esophageal carcinoma \\
\hline RT-qPCR & Real-time quantitative polymerase chain reaction \\
\hline ceRNA & Long nocoding RNA \\
\hline ChIP & Chrompeting endogenous RNA \\
\hline EdU & Ethynyl deoxyuridine \\
\hline RIP & RNA immunoprecipitation \\
\hline FISH & RNA fluorescent in situ hybridization \\
\hline ACAA2 & Acetyl CoA acyltransferase 2 \\
\hline CAT & Catalase \\
\hline ALOX12B & Arachidonate 12-lipoxygenase, 12R-type \\
\hline ChIRP & Comprehensive identification of RNA binding proteins \\
\hline TCGA & The Cancer Genome Atlas \\
\hline
\end{tabular}

\section{Declarations}

\section{Ethics approval and consent to participate}

This study was approved by the Southeast University Affiliated Zhongda Hospital Ethics Committee, and it was conducted in compliance with the Helsinki Declaration. All eligible participants have signed written informed consent for publication. All animal procedures were approved by the Animal Care and Use Committee of Southeast University.

\section{Consent for publication}

Not applicable

\section{Availability of data and materials}

The datasets used and/or analyzed during the current study are available from the corresponding author on reasonable request

\section{Competing Interests}

The authors declare no competing financial interests. 


\section{Funding}

This work was supported by the National Natural Science Foundation of China (No. 81872588, 82073516).

\section{Authors' contributions}

$\mathrm{HZ}$ performed the experiments, analyzed the data, prepared the manuscript and approved the final draft. ECP, YZ, CZ, QWL, QL, JJZ collected tissue specimens and information of patients. LHY, SZW, RL and YPP guided this research. All authors read and approved the final manuscript.

\section{Acknowledgements}

Not applicable

\section{Author information}

Affiliations

${ }^{1}$ Key Laboratory of Environmental Medicine Engineering, Ministry of Education, School of Public Health, Southeast University, Nanjing 210009, People's Republic of China

Hu Zhang, Ying Zhang, Chao Zhao, Qiwei Liu, Qiang Lu, Jingjing Zhou, Shizhi Wang, Ran Liu, Yuepu Pu \& Lihong Yin

\section{${ }^{2}$ Huaian Center for Disease Control and Prevention, Huaian 223001, People's Republic of China Enchun Pan}

\section{References}

1. Bray F, Ferlay J, Soerjomataram I, Siegel RL, Torre LA, Jemal A. Global cancer statistics 2018 : GLOBOCAN estimates of incidence and mortality worldwide for 36 cancers in 185 countries. CA Cancer J Clin. 2018;68(6):394-424.

2. Chen W, Zheng R, Baade PD, Zhang S, Zeng H, Bray F, et al. Cancer statistics in China, 2015. CA Cancer J Clin. 2016;66(2):115-32.

3. Abnet CC, Arnold M, Wei WQ. Epidemiology of Esophageal Squamous Cell Carcinoma. Gastroenterology. 2018;154(2):360-73.

4. Yang J, Liu X, Cao S, Dong X, Rao S, Cai K. Understanding Esophageal Cancer: The Challenges and Opportunities for the Next Decade. Front Oncol. 2020;10:1727.

5. Guan X, Liu C, Zhou T, Ma Z, Zhang C, Wang B, et al. Survival and prognostic factors of patients with esophageal fistula in advanced esophageal squamous cell carcinoma. Biosci Rep. 2020; 40(1). 
6. Shen S, Li K, Liu Y, Liu X, Liu B, Ba Y, et al. Silencing IncRNA AGAP2-AS1 Upregulates miR-195-5p to Repress Migration and Invasion of EC Cells via the Decrease of FOSL1 Expression. Mol Ther Nucleic Acids. 2020;20:331-44.

7. Qian X, Zhao J, Yeung PY, Zhang QC, Kwok CK. Revealing IncRNA Structures and Interactions by Sequencing-Based Approaches. Trends Biochem Sci. 2019;44(1):33-52.

8. Sallam T, Sandhu J, Tontonoz P. Long Noncoding RNA Discovery in Cardiovascular Disease: Decoding Form to Function. Circ Res. 2018;122(1):155-66.

9. McDonel P, Guttman M. Approaches for Understanding the Mechanisms of Long Noncoding RNA Regulation of Gene Expression. Cold Spring Harb Perspect Biol. 2019; 11(12).

10. Lorenzi L, Avila Cobos F, Decock A, Everaert C, Helsmoortel H, Lefever S, et al. Long noncoding RNA expression profiling in cancer: Challenges and opportunities. Genes Chromosomes Cancer. 2019;58(4):191-9.

11. Tian W, Jiang C, Huang Z, Xu D, Zheng S. Comprehensive analysis of dysregulated IncRNAs, miRNAs and mRNAs with associated ceRNA network in esophageal squamous cell carcinoma. Gene. 2019;696:206-18.

12. Wang X, Li M, Wang Z, Han S, Tang X, Ge Y, et al. Silencing of long noncoding RNA MALAT1 by miR101 and miR-217 inhibits proliferation, migration, and invasion of esophageal squamous cell carcinoma cells. J Biol Chem. 2015;290(7):3925-35.

13. Gao H, Wang T, Zhang P, Shang M, Gao Z, Yang F, et al. Linc-ROR regulates apoptosis in esophageal squamous cell carcinoma via modulation of p53 ubiquitination by targeting miR-204-5p/MDM2. J Cell Physiol. 2020;235(2):2325-35.

14. Liang Y, Chen XD, Wu YY, Li J, Zhang SX, Wang K, et al. LncRNA CASC9 promotes esophageal squamous cell carcinoma metastasis through upregulating LAMC2 expression by interacting with the CREB-binding protein. Cell Death Differ (Article). 2018;25(11):1980-95.

15. Shi W, Wang Q, Bian Y, Fan Y, Zhou Y, Feng T, et al. Long noncoding RNA PANDA promotes esophageal squamous carcinoma cell progress by dissociating from NF-YA but interact with SAFA. Pathol Res Pract. 2019;215(10):152604.

16. Gong Z, Li J, Cang P, Jiang H, Liang J, Hou Y. RPL34-AS1 functions as tumor suppressive IncRNA in esophageal cancer. Biomedicine \& Pharmacotherapy. 2019; 120 (Unsp 109440).

17. Wei H, Yang Z, Lin B. Overexpression of long non coding RNA CA3-AS1 suppresses proliferation, invasion and promotes apoptosis via miRNA-93/PTEN axis in colorectal cancer. Gene. 2019;687:915.

18. Zhao J, Liu Y, Zhang W, Zhou Z, Wu J, Cui P, et al. Long non-coding RNA Linc00152 is involved in cell cycle arrest, apoptosis, epithelial to mesenchymal transition, cell migration and invasion in gastric cancer. Cell Cycle. 2015;14(19):3112-23.

19. Ji L, Fan X, Zhou F, Gu J, Deng X. IncRNA RPL34-AS1 inhibits cell proliferation and invasion while promoting apoptosis by competitively binding miR-3663-3p/RGS4 in papillary thyroid cancer. J Cell Physiol. 2020;235(4):3669-78. 
20. Miao Y, Sui J, Zhang Y, Yin L. Development of a novel signature of long noncoding RNAs as a prognostic biomarker for esophageal cancer. BioRxiv. 2018; e-pub ahead of print 13 Octorber 2018; doi.org/10.1101/441568.

21. Gao ZK, Liu H, Shi YJ, Yin LH, Zhu Y, Liu R. Identification of Cancer Stem Cell Molecular Markers and Effects of hsa-miR-21-3p on Stemness in Esophageal Squamous Cell Carcinoma. Cancers. 2019; 11.

22. Zhang Y, Miao Y, Shang MH, Liu MX, Liu R, Pan EC, et al. LincRNA-p21 leads to G1 arrest by p53 pathway in esophageal squamous cell carcinoma. Cancer Manag Res. 2019;11:6201-14.

23. Chu C, Chang HY. ChIRP-MS: RNA-Directed Proteomic Discovery. Methods Mol Biol. 2018;1861:3745.

24. Zheng YX, Yin HY, Boeglin WE, Elias PM, Crumrine D, Beier DR, et al. Lipoxygenases Mediate the Effect of Essential Fatty Acid in Skin Barrier Formation A PROPOSED ROLE IN RELEASING OMEGAHYDROXYCERAMIDE FOR CONSTRUCTION OF THE CORNEOCYTE LIPID ENVELOPE. J Biol Chem. 2011;286(27):24046-56.

25. Takeuchi A, Miyamoto T, Yamaji K, Masuho Y, Hayashi M, Hayashi H, et al. A Human ErythrocyteDerived Growth-Promoting Factor with a Wide Target-Cell Spectrum - Identification as Catalase. Cancer Res. 1995;55(7):1586-9.

26. Lorenzi L, Cobos FA, Decock A, Everaert C, Helsmoortel H, Lefever S, et al. Long noncoding RNA expression profiling in cancer: Challenges and opportunities. Gene Chromosome Canc. 2019;58(4):191-9.

27. Wang C, Duan Y, Duan G, Wang Q, Zhang K, Deng X, et al. Stress Induces Dynamic, CytotoxicityAntagonizing TDP-43 Nuclear Bodies via Paraspeckle LncRNA NEAT1-Mediated Liquid-Liquid Phase Separation. Mol Cell. 2020;79:443-58. e447.

28. Latge G, Poulet C, Bours V, Josse C, Jerusalem G. Natural Antisense Transcripts: Molecular Mechanisms and Implications in Breast Cancers. Int J Mol Sci. 2018; 19(1).

29. Talebi A, Masoodi M, Mirzaei A, Mehrad-Majd H, Azizpour M, Akbari A. Biological and clinical relevance of metastasis-associated long noncoding RNAs in esophageal squamous cell carcinoma: A systematic review. J Cell Physiol. 2020;235(2):848-68.

30. Huang Y, Guo Q, Ding XP, Wang XT. Mechanism of long noncoding RNAs as transcriptional regulators in cancer. Rna Biol. 2020;17:1680-92.

31. Engreitz JM, Ollikainen N, Guttman M. Long non-coding RNAs: spatial amplifiers that control nuclear structure and gene expression. Nat Rev Mol Cell Bio. 2016;17(12):756-70.

32. Zhang EB, He XZ, Zhang CG, Su J, Lu XY, Si XX, et al. A novel long noncoding RNA HOXC-AS3 mediates tumorigenesis of gastric cancer by binding to YBX1. Genome Biol. 2018;19(1):154.

33. Farrelly LA, Thompson RE, Zhao S, Lepack AE, Lyu Y, Bhanu NV, et al. Histone serotonylation is a permissive modification that enhances TFIID binding to H3K4me3. Nature. 2019;567(7749):535-9.

34. Liu DS, Zhang H, Cong JC, Cui MM, Ma MX, Zhang FY, et al. H3K27 acetylation-induced IncRNA EIF3J-AS1 improved proliferation and impeded apoptosis of colorectal cancer through miR3163/YAP1 axis. J Cell Biochem. 2020;121(2):1923-33. 
35. Hu YP, Jin YP, Wu XS, Yang Y, Li YS, Li HF, et al. LncRNA-HGBC stabilized by HuR promotes gallbladder cancer progression by regulating miR-502-3p/SET/AKT axis. Mol Cancer. 2019;18(1):167.

36. Tay Y, Rinn J, Pandolfı PP. The multilayered complexity of ceRNA crosstalk and competition. Nature. 2014;505(7483):344-52.

37. Kiema TR, Harijan RK, Strozyk M, Fukao T, Alexson SEH, Wierenga RK. The crystal structure of human mitochondrial 3-ketoacyl-CoA thiolase $(T 1)$ : insight into the reaction mechanism of its thiolase and thioesterase activities. Acta Crystallogr D. 2014;70:3212-25.

38. Gao YF, Liu JY, Mao XY, He ZW, Zhu T, Wang ZB, et al. LncRNA FOXD1-AS1 acts as a potential oncogenic biomarker in glioma. Cns Neurosci Ther. 2020;26(1):66-75.

39. Muret K, Desert C, Lagoutte L, Boutin M, Gondret F, Zerjal T, et al. Long noncoding RNAs in lipid metabolism: literature review and conservation analysis across species. Bmc Genomics. 2019;20(1):882.

40. Lin W, Zhou Q, Wang CQ, Zhu L, Bi C, Zhang S, et al. LncRNAs regulate metabolism in cancer. Int J Biol Sci. 2020;16(7):1194-206.

41. Aryal B, Suarez Y. Non-coding RNA regulation of endothelial and macrophage functions during atherosclerosis. Vasc Pharmacol. 2019;114:64-75.

\section{Figures}



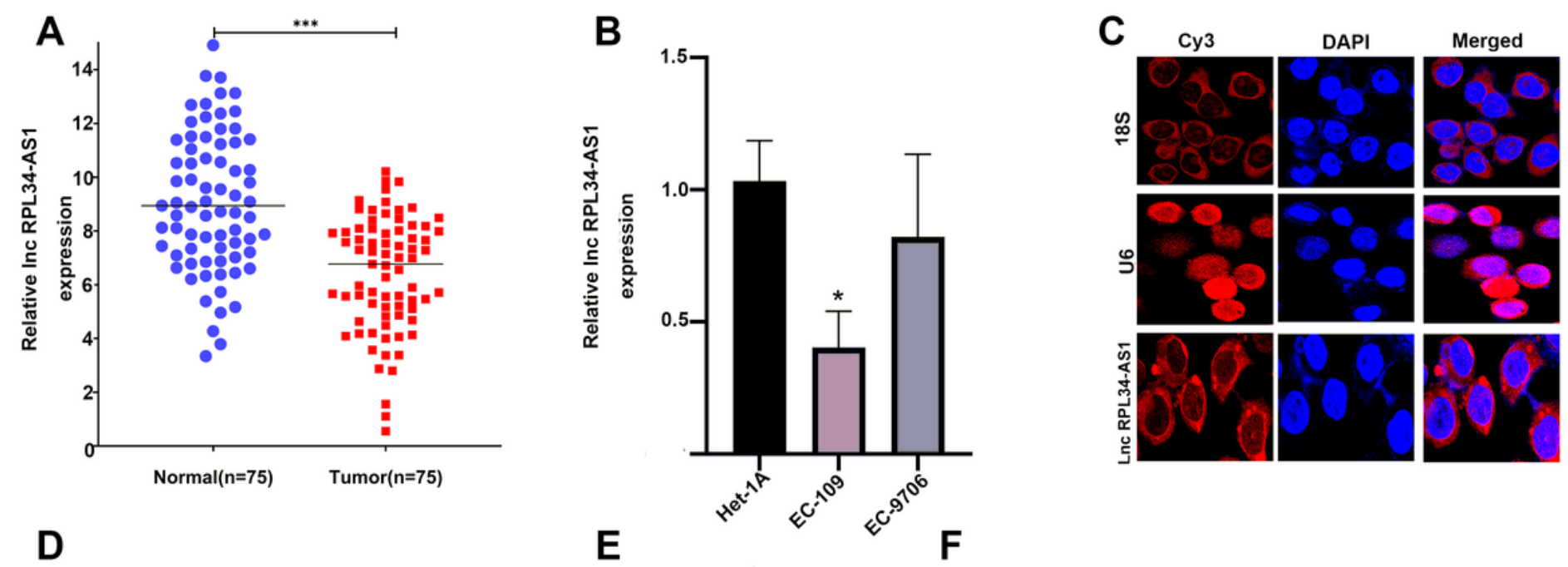

D

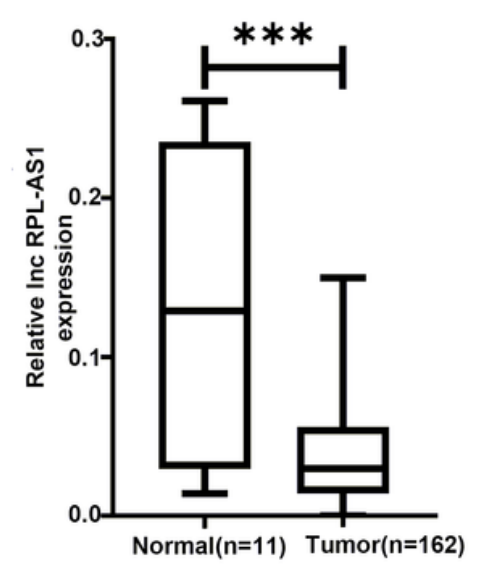

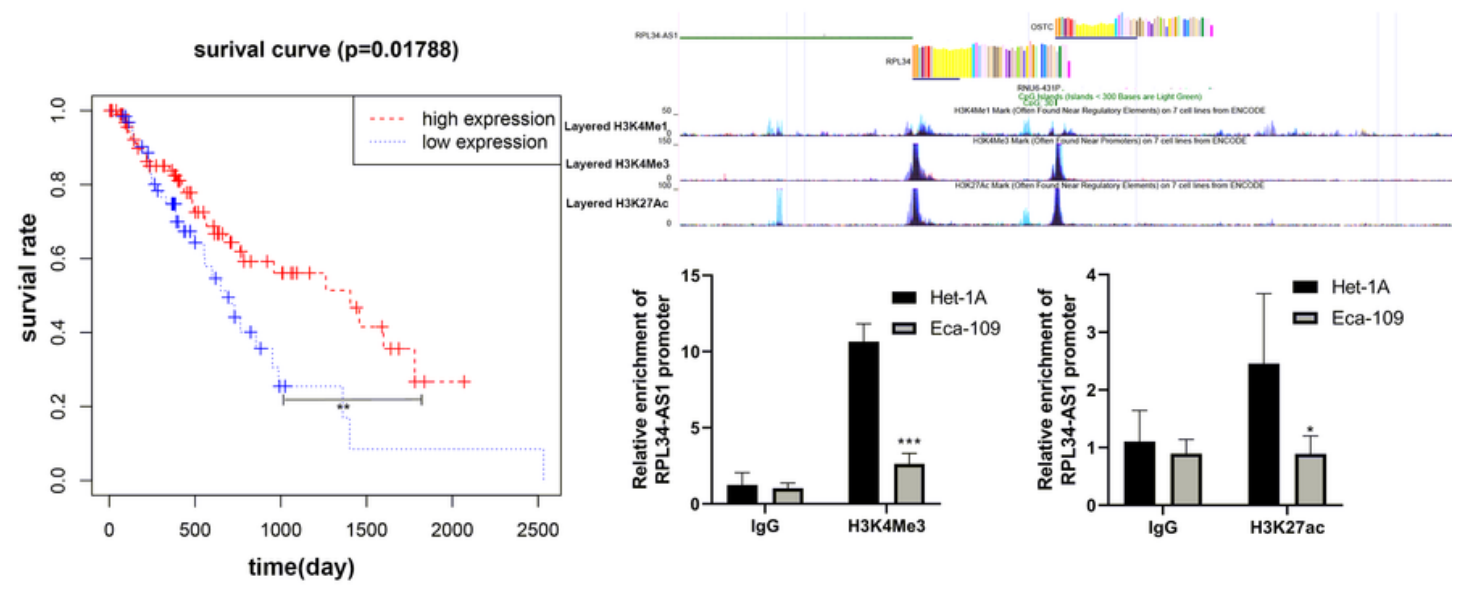

Figure 1

Identification of RPL34-AS1 and expression of RPL34-AS1 in ESCC tissues and its clinical parameters and gain of H3K4me3 and H3K27 acetylation could reduce activated RPL34-AS1 in ESCC. a. Relative expression of RPL34-AS1 in ESCC and matched adjacent normal tissues was detected by RT-qPCR $(\mathrm{n}=$ 75). b. Relative expression of RPL34-AS1 in cell lines was determined by RT-qPCR. c. Using Single Molecule IncRNA Fluorescent in Situ Hybridization (IncRNA-FISH) to fix a position on RPL34-AS1. d. RPL34-AS1 expression in ESCC tissues $(n=162)$ compared with noncancerous tissues $(n=11)$ analyzed using the TCGA database. e. Kaplan-Meier survival curves of ESCC patients with low and high RPL34-AS1 expression. Using median RPL34-AS1 value as a cutoff. f. The UCSC Genome Bioinformatics Site (http://genome.ucsc.edu/) showed high enrichment of H3K4me3 and H3K27ac at the promoter of RPL34AS1. ChIP assays detected the level of H3K4me3 and H3K27ac at the promoter of RPL34-AS1 in ESCC cells. Data were showed as mean $\pm \mathrm{SD}$. ${ }^{\star} \mathrm{P}<0.05,{ }^{\star *} \mathrm{P}<0.01,{ }^{*} * \mathrm{P}<0.001$. 

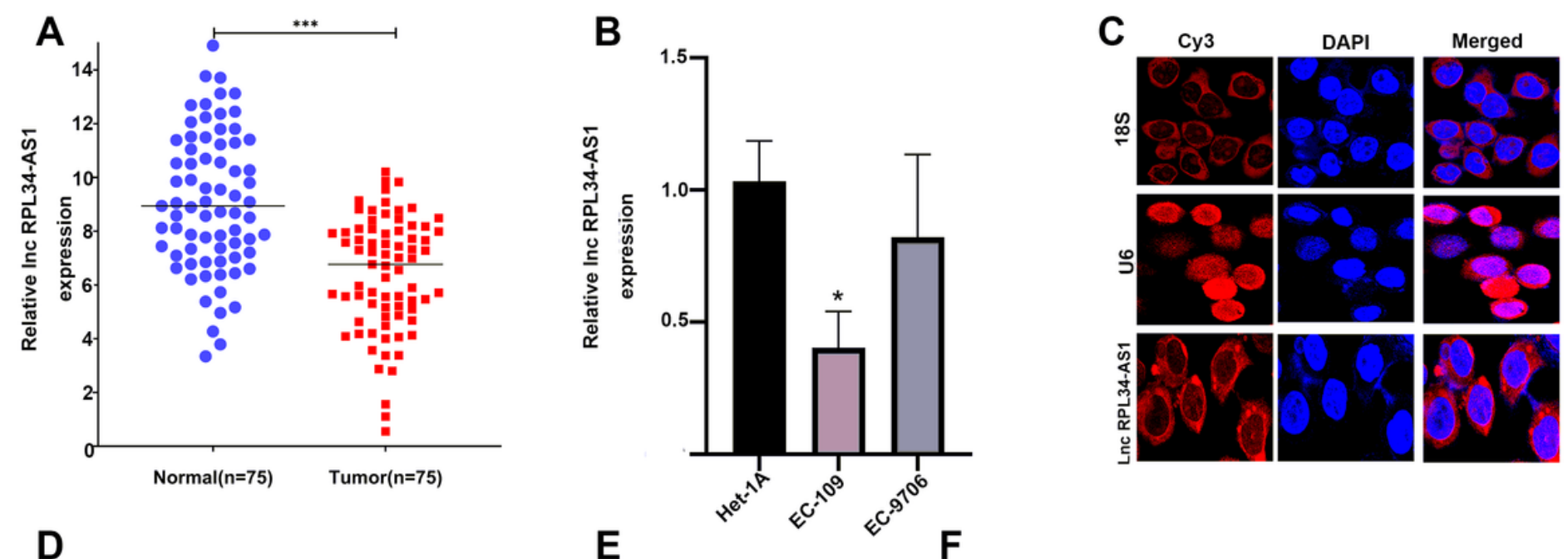

D

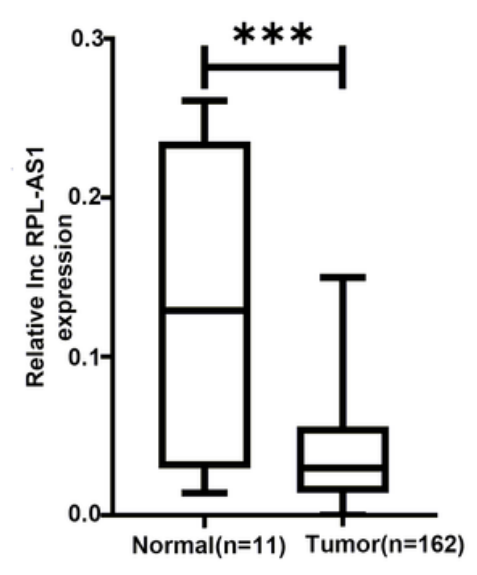

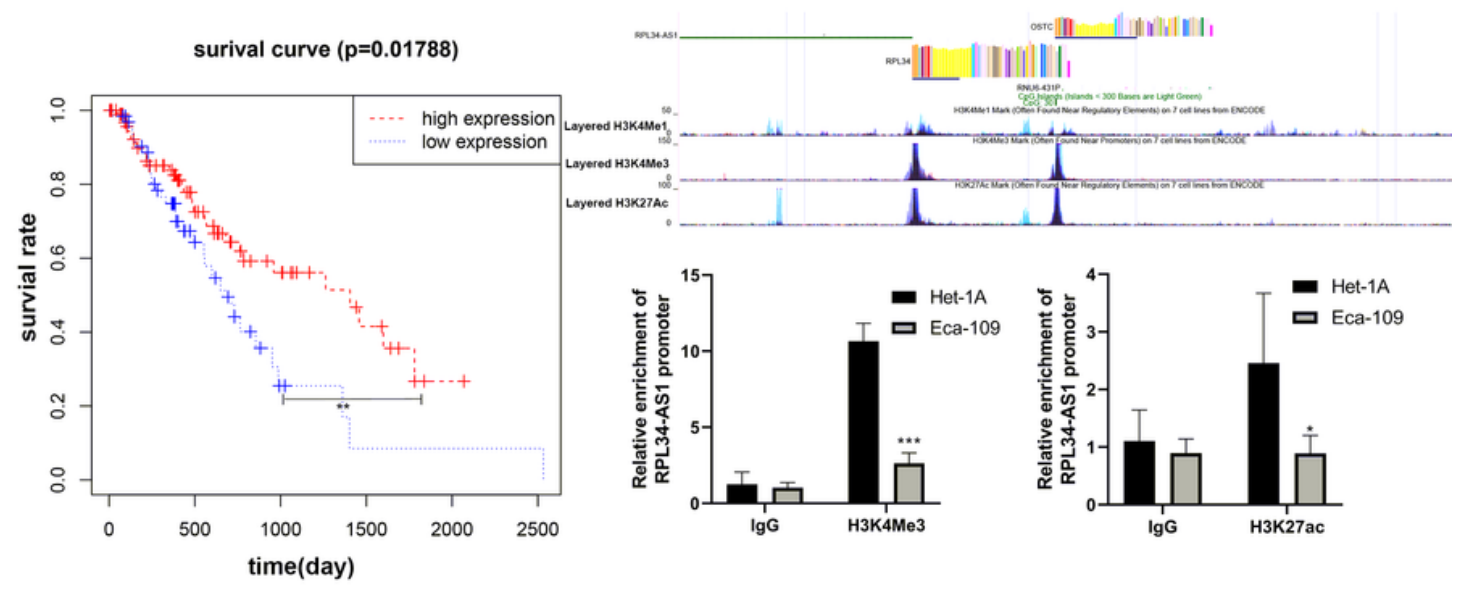

Figure 1

Identification of RPL34-AS1 and expression of RPL34-AS1 in ESCC tissues and its clinical parameters and gain of H3K4me3 and H3K27 acetylation could reduce activated RPL34-AS1 in ESCC. a. Relative expression of RPL34-AS1 in ESCC and matched adjacent normal tissues was detected by RT-qPCR $(\mathrm{n}=$ 75). b. Relative expression of RPL34-AS1 in cell lines was determined by RT-qPCR. c. Using Single Molecule IncRNA Fluorescent in Situ Hybridization (IncRNA-FISH) to fix a position on RPL34-AS1. d. RPL34-AS1 expression in ESCC tissues $(n=162)$ compared with noncancerous tissues $(n=11)$ analyzed using the TCGA database. e. Kaplan-Meier survival curves of ESCC patients with low and high RPL34-AS1 expression. Using median RPL34-AS1 value as a cutoff. f. The UCSC Genome Bioinformatics Site (http://genome.ucsc.edu/) showed high enrichment of H3K4me3 and H3K27ac at the promoter of RPL34AS1. ChIP assays detected the level of H3K4me3 and H3K27ac at the promoter of RPL34-AS1 in ESCC cells. Data were showed as mean $\pm \mathrm{SD}$. ${ }^{\star} \mathrm{P}<0.05,{ }^{\star *} \mathrm{P}<0.01,{ }^{*} * \mathrm{P}<0.001$. 

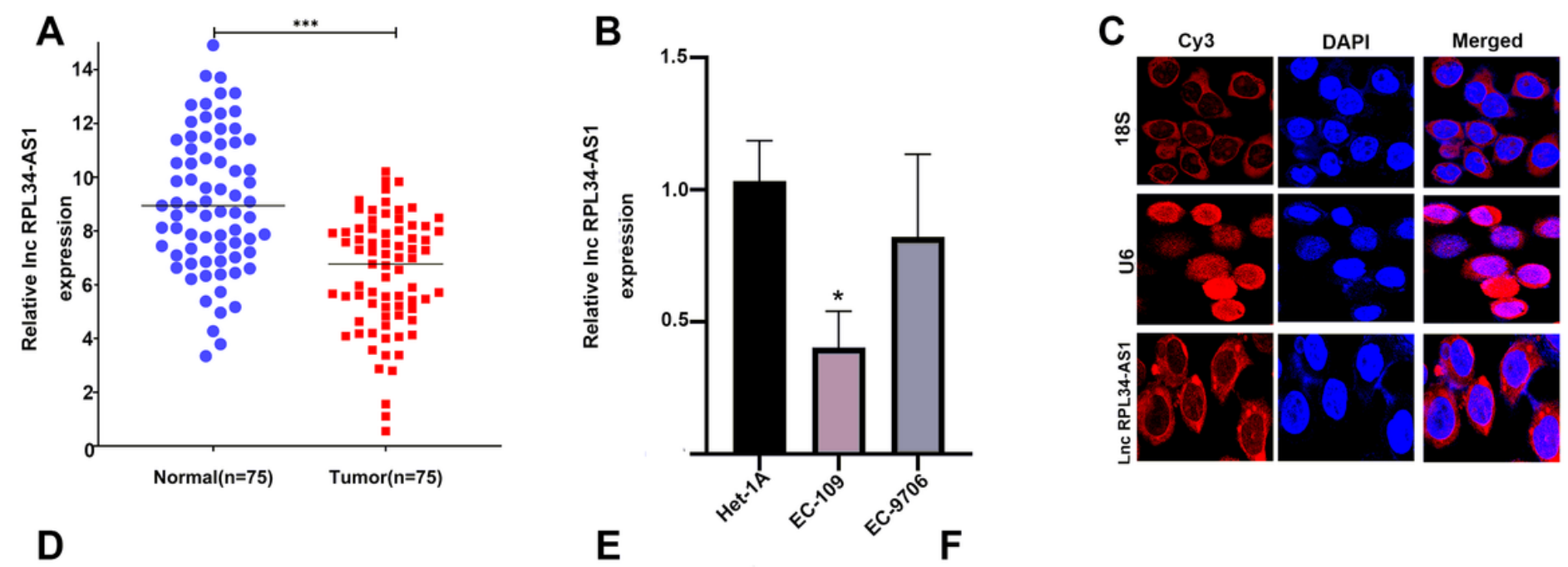

D

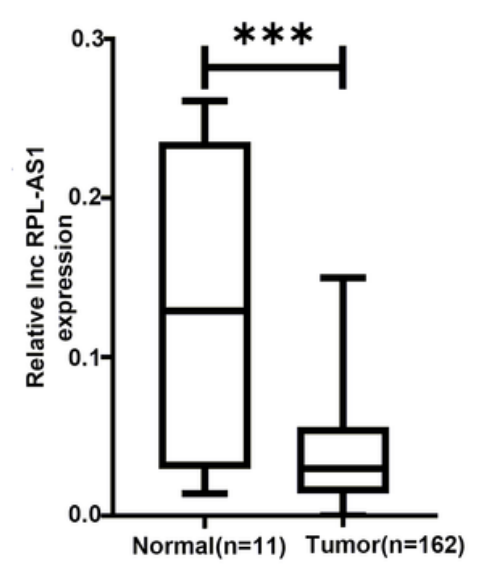

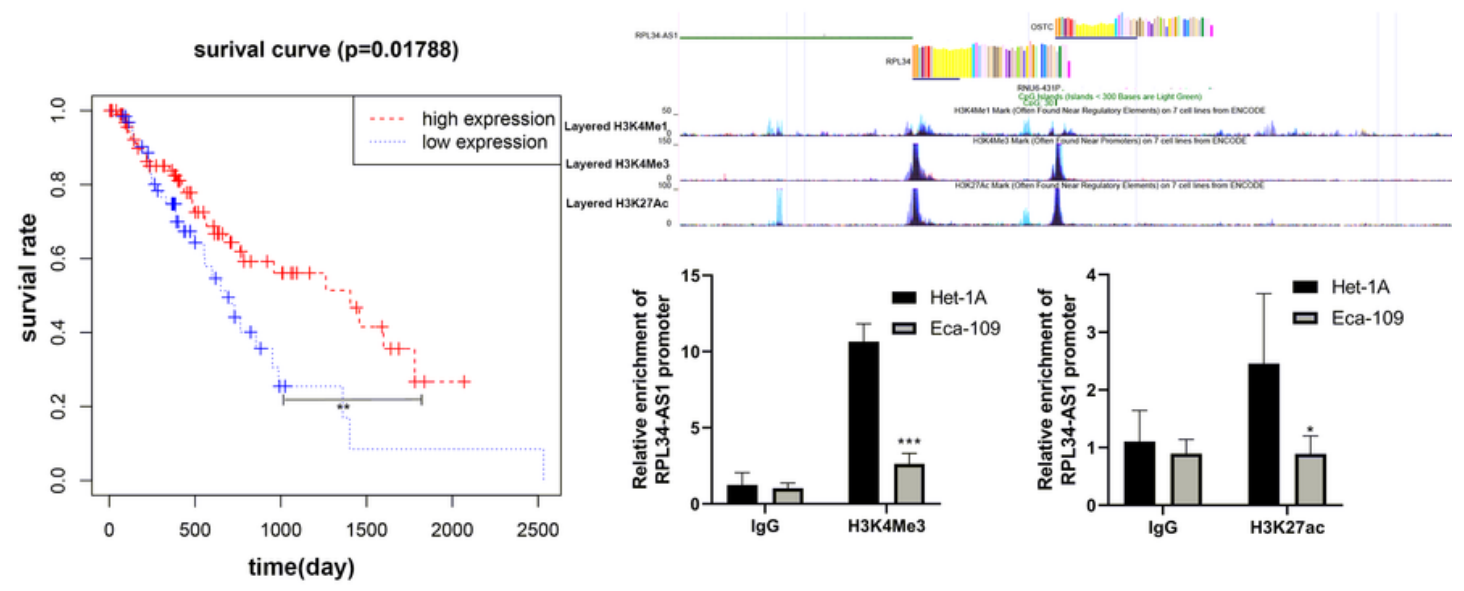

Figure 1

Identification of RPL34-AS1 and expression of RPL34-AS1 in ESCC tissues and its clinical parameters and gain of H3K4me3 and H3K27 acetylation could reduce activated RPL34-AS1 in ESCC. a. Relative expression of RPL34-AS1 in ESCC and matched adjacent normal tissues was detected by RT-qPCR $(\mathrm{n}=$ 75). b. Relative expression of RPL34-AS1 in cell lines was determined by RT-qPCR. c. Using Single Molecule IncRNA Fluorescent in Situ Hybridization (IncRNA-FISH) to fix a position on RPL34-AS1. d. RPL34-AS1 expression in ESCC tissues $(n=162)$ compared with noncancerous tissues $(n=11)$ analyzed using the TCGA database. e. Kaplan-Meier survival curves of ESCC patients with low and high RPL34-AS1 expression. Using median RPL34-AS1 value as a cutoff. f. The UCSC Genome Bioinformatics Site (http://genome.ucsc.edu/) showed high enrichment of H3K4me3 and H3K27ac at the promoter of RPL34AS1. ChIP assays detected the level of H3K4me3 and H3K27ac at the promoter of RPL34-AS1 in ESCC cells. Data were showed as mean $\pm \mathrm{SD}$. ${ }^{\star} \mathrm{P}<0.05,{ }^{\star *} \mathrm{P}<0.01,{ }^{*} * \mathrm{P}<0.001$. 


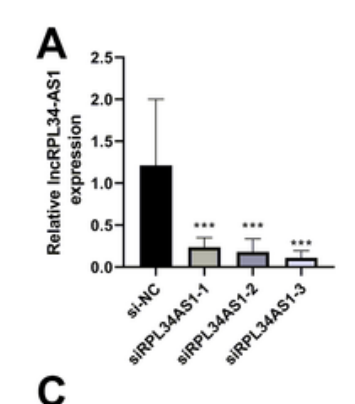

C
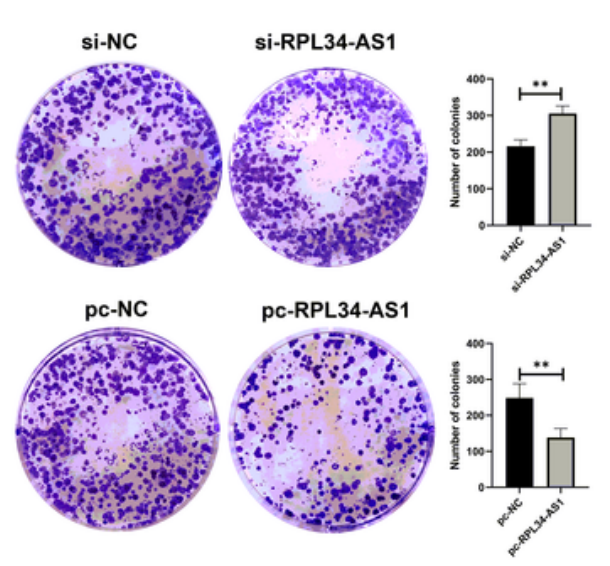

B
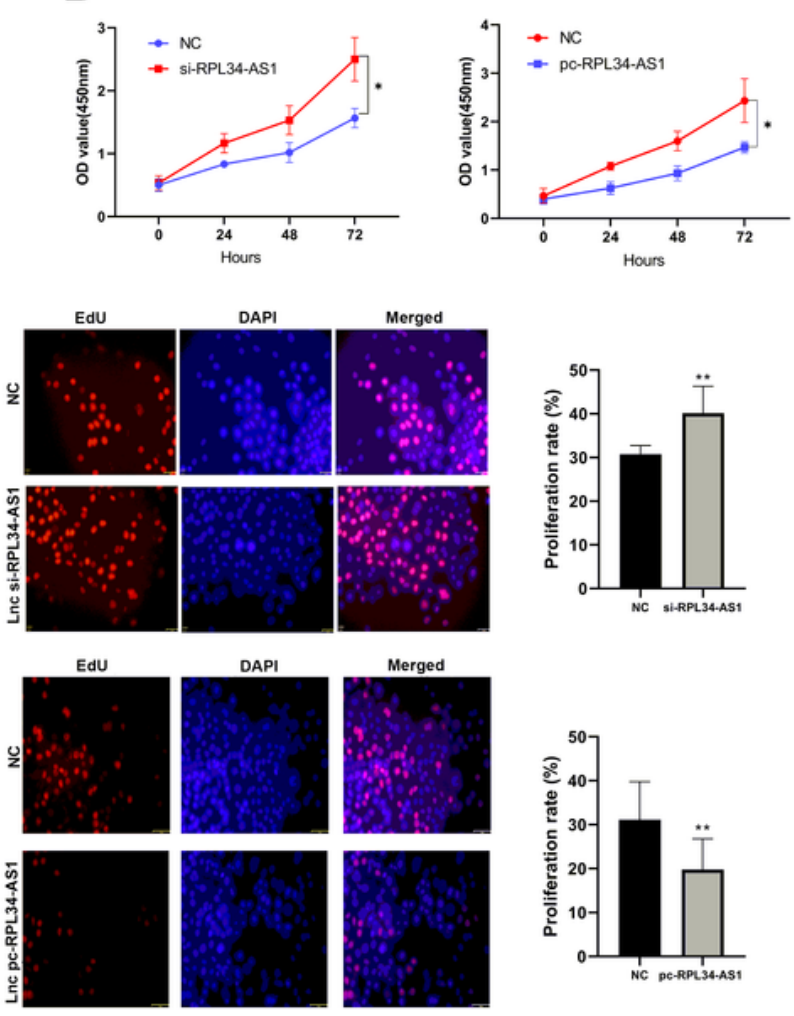

E

$\mathbf{F}$

G
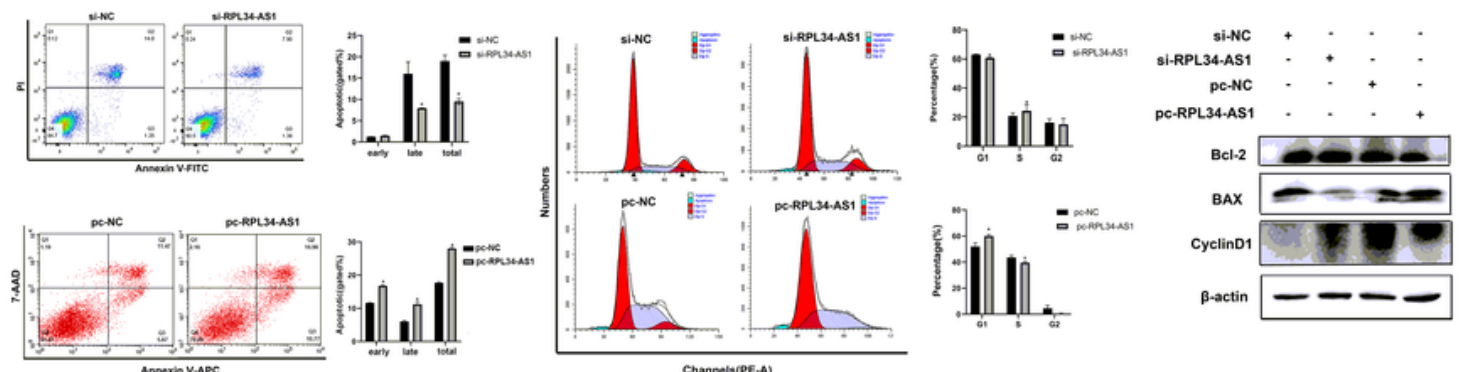

H
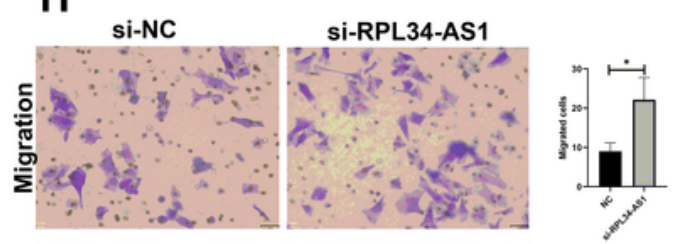

I NC
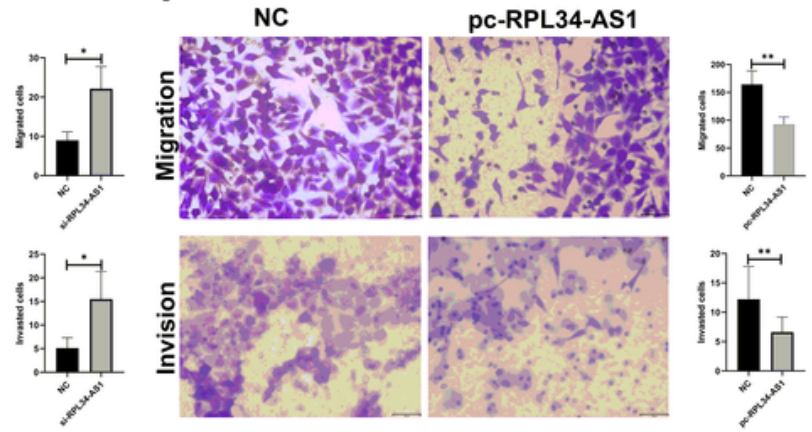

\section{Figure 2}

LncRPL34-AS1 suppresses ESCC cell proliferation, cell-cycle progression, migration and invasion and promotes cell apoptosis in vitro. a. LncRPL34-AS1 expression level was detected in EC109 cells by RTqPCR after up-regulated and down-regulated transfection. b-d. CCK-8, colony formation and EdU assays were performed to determine the ability of proliferation in EC109 cells transfected with si-Inc or NC and transfected with pc-Inc or NC. Scale bar, $50 \mu \mathrm{m}$. e, f. Flow cytometry was performed to determine the 
effect of IncRPL34-AS1 on cell apoptosis and cell cycle by flow cytometry analysis. g. The levels of apoptosis protein Bcl-2, BAX and cycle protein Cyclin D1 expression were detected by western blot. h, i. Cell migratory and invasive capabilities were assessed by transwell assays in EC109 cells transfected with si-Inc or NC and transfected with pc-Inc or NC. Scale bar, $50 \mu \mathrm{m}$. Data were showed as mean $\pm \mathrm{SD}$. *P $<0.05, * * \mathrm{P}<0.01, * * * \mathrm{P}<0.001$.
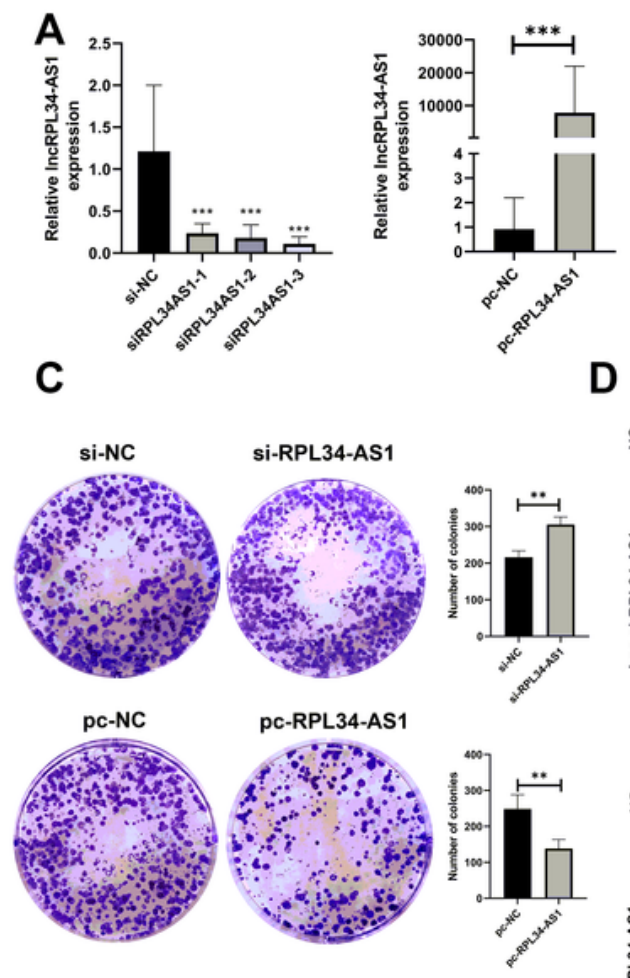

D
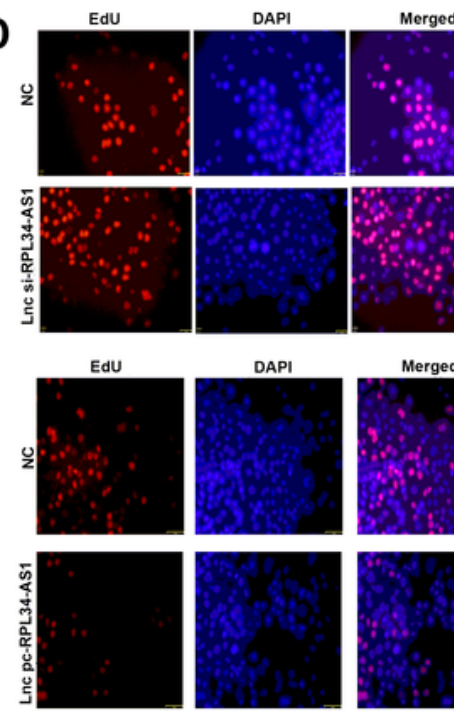

$\mathbf{F}$
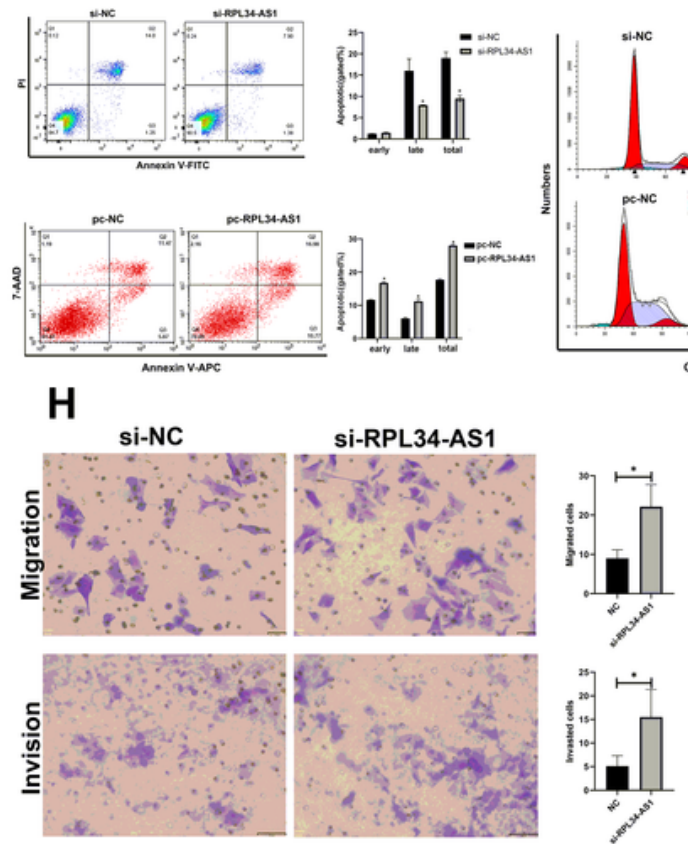
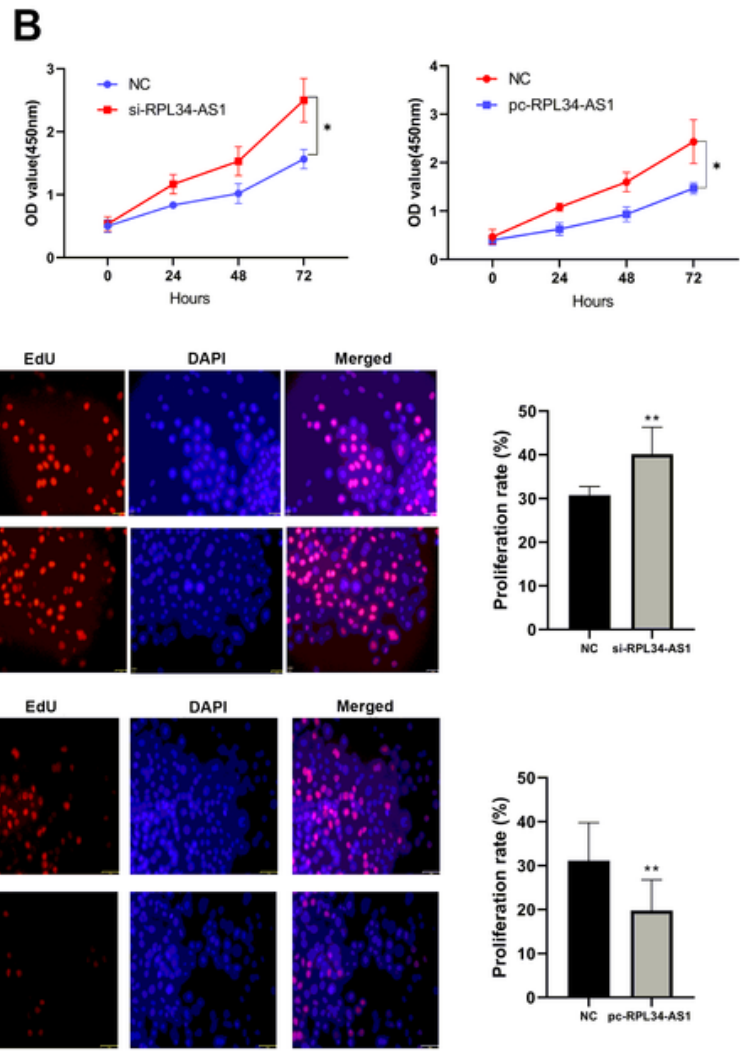

Merged
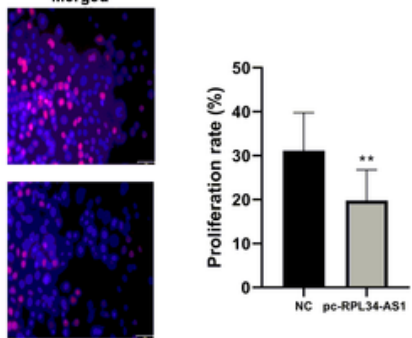

G

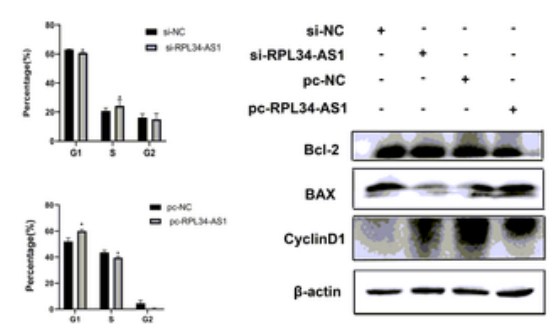

I
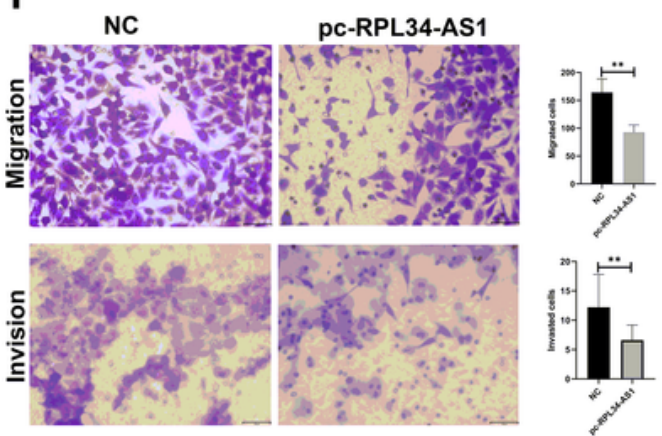

Figure 2 
LncRPL34-AS1 suppresses ESCC cell proliferation, cell-cycle progression, migration and invasion and promotes cell apoptosis in vitro. a. LncRPL34-AS1 expression level was detected in EC109 cells by RTqPCR after up-regulated and down-regulated transfection. b-d. CCK-8, colony formation and EdU assays were performed to determine the ability of proliferation in EC109 cells transfected with si-Inc or NC and transfected with pc-Inc or NC. Scale bar, $50 \mu \mathrm{m}$. e, f. Flow cytometry was performed to determine the effect of IncRPL34-AS1 on cell apoptosis and cell cycle by flow cytometry analysis. g. The levels of apoptosis protein Bcl-2, BAX and cycle protein Cyclin D1 expression were detected by western blot. h, i. Cell migratory and invasive capabilities were assessed by transwell assays in EC109 cells transfected with si-Inc or NC and transfected with pc-Inc or NC. Scale bar, $50 \mu \mathrm{m}$. Data were showed as mean $\pm \mathrm{SD}$. *P $<0.05, \star \star P<0.01, \star \star \star P P<0.001$. 


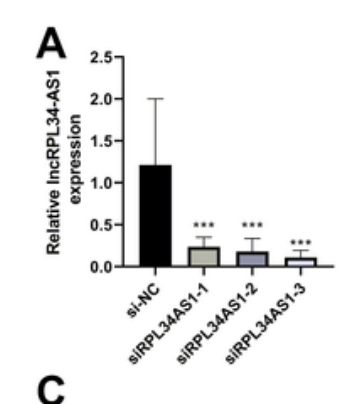

C
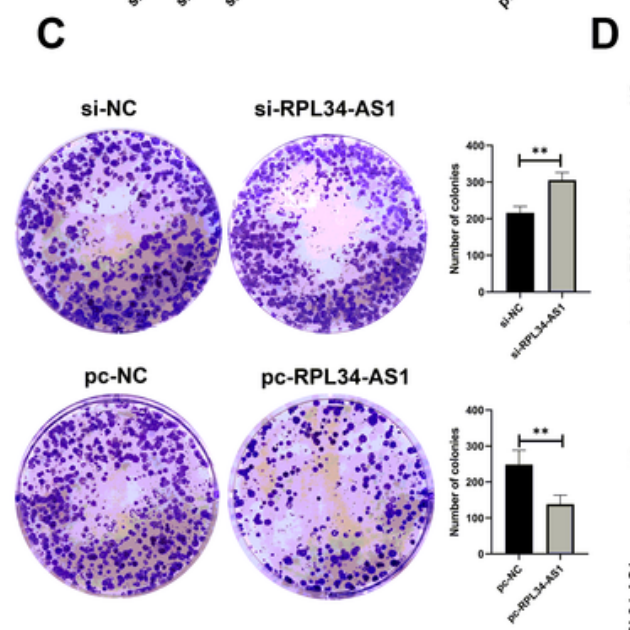

B
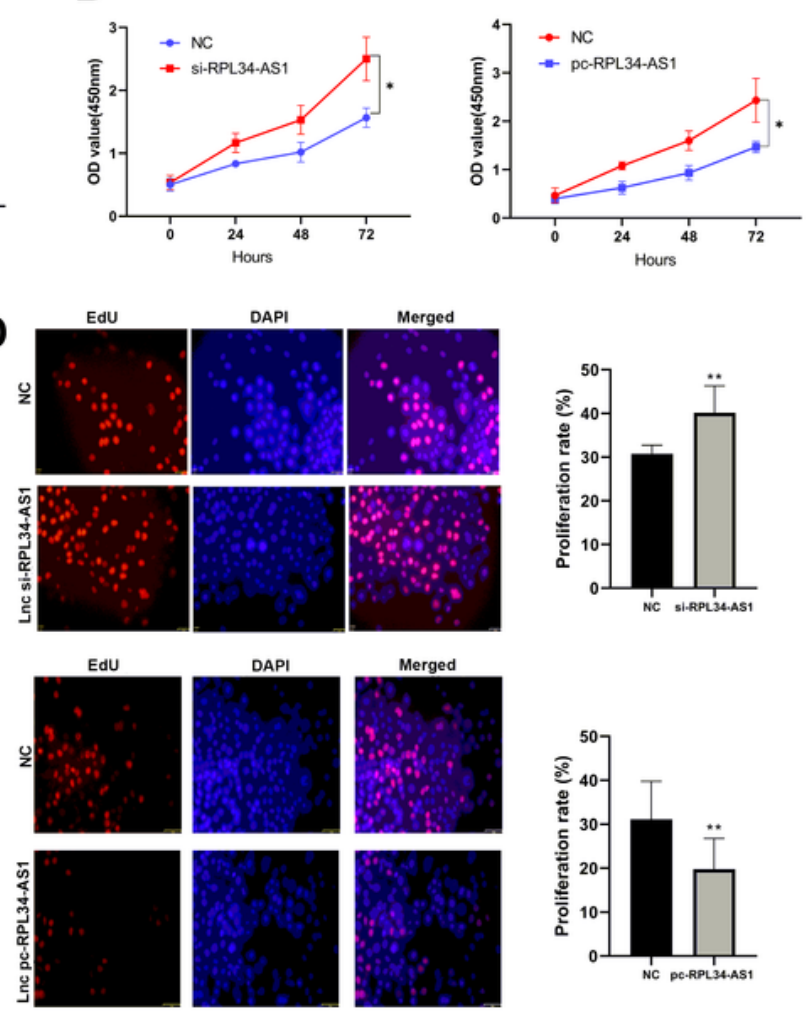

E

$\mathbf{F}$

G

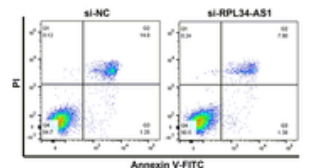<smiles>[AlH2]</smiles>
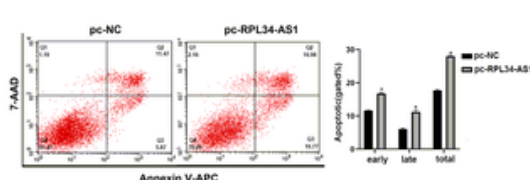

H
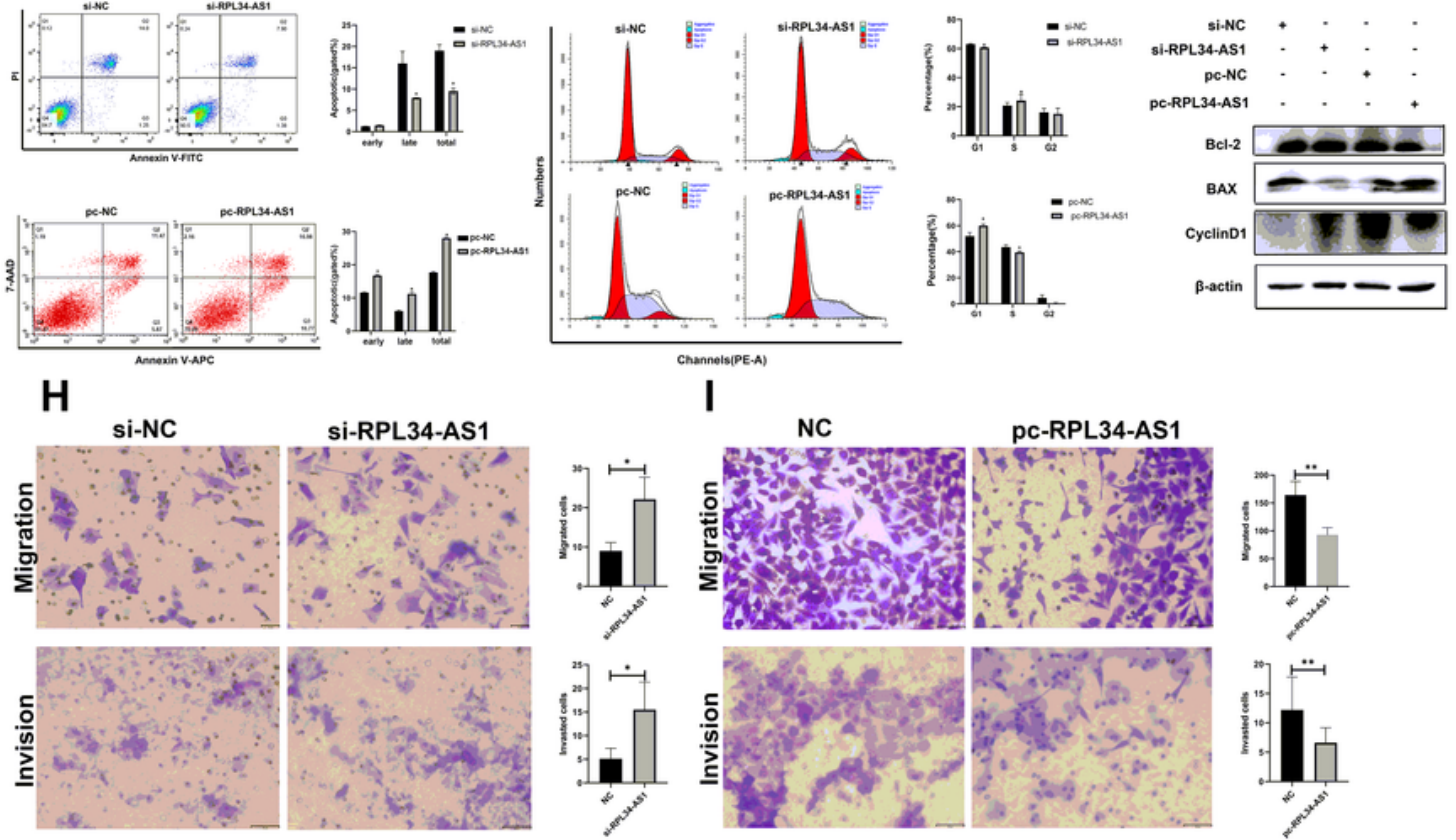

I NC
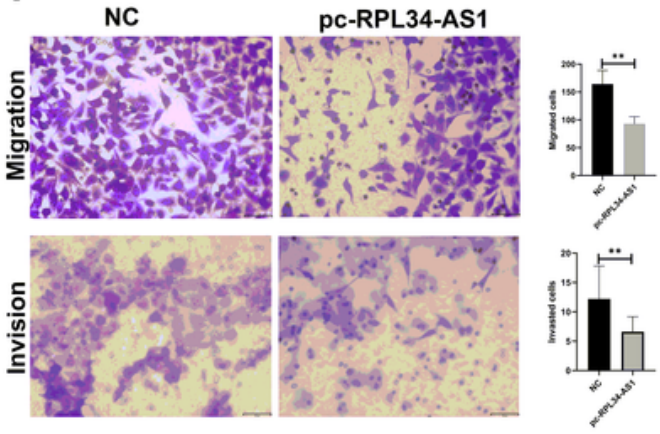

Figure 2

LncRPL34-AS1 suppresses ESCC cell proliferation, cell-cycle progression, migration and invasion and promotes cell apoptosis in vitro. a. LncRPL34-AS1 expression level was detected in EC109 cells by RTqPCR after up-regulated and down-regulated transfection. b-d. CCK-8, colony formation and EdU assays were performed to determine the ability of proliferation in EC109 cells transfected with si-Inc or NC and transfected with pc-Inc or NC. Scale bar, $50 \mu \mathrm{m}$. e, f. Flow cytometry was performed to determine the 
effect of IncRPL34-AS1 on cell apoptosis and cell cycle by flow cytometry analysis. g. The levels of apoptosis protein Bcl-2, BAX and cycle protein Cyclin D1 expression were detected by western blot. h, i. Cell migratory and invasive capabilities were assessed by transwell assays in EC109 cells transfected with si-Inc or NC and transfected with pc-Inc or NC. Scale bar, $50 \mu \mathrm{m}$. Data were showed as mean $\pm \mathrm{SD}$. ${ }^{\mathrm{P}}$ $<0.05, * * \mathrm{P}<0.01, * * * \mathrm{P}<0.001$.

A

pc-NC

pc-RPL34-AS1

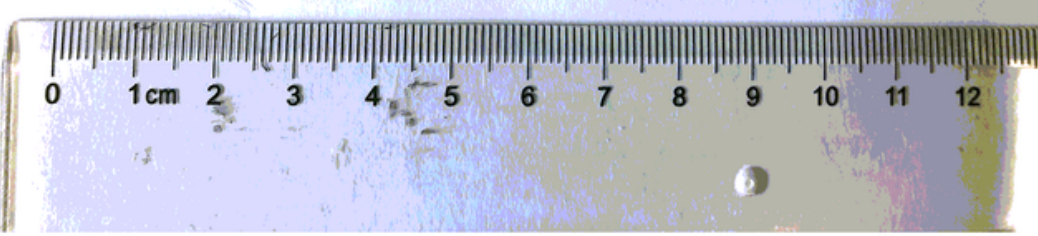

C

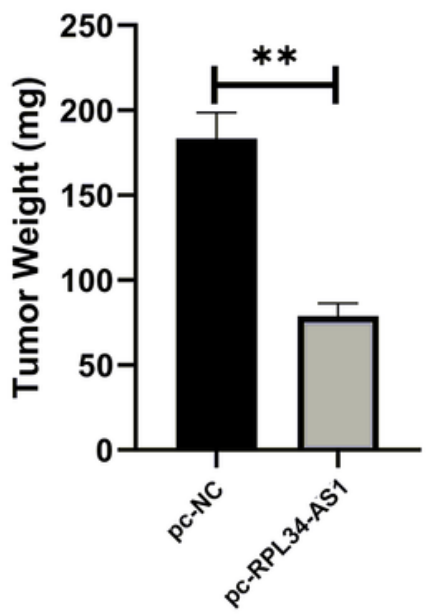

$\mathbf{F}$

pc-NC
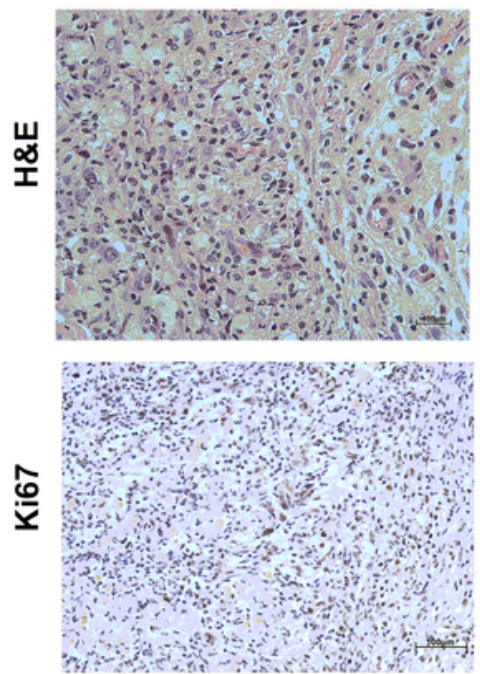

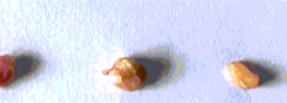

$\rightarrow$

D

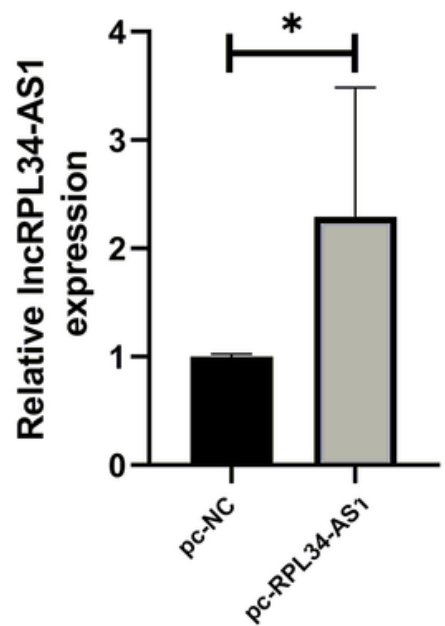

pc-RPL34-AS1
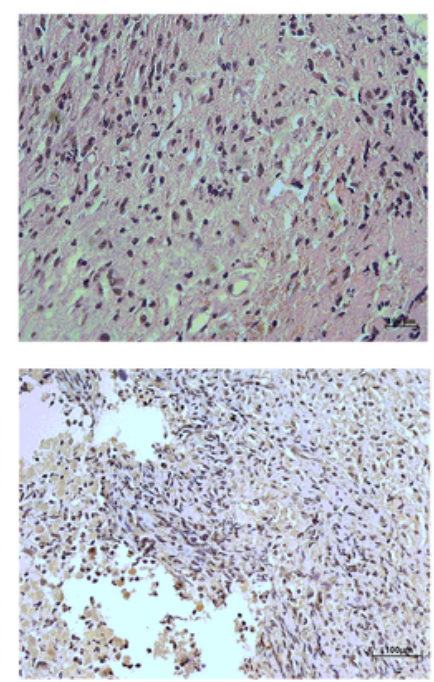

B

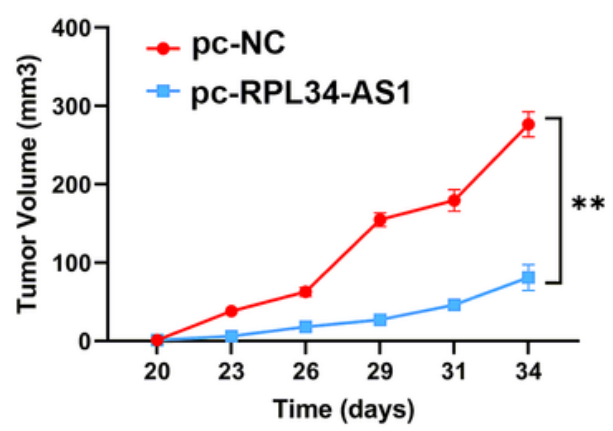

E

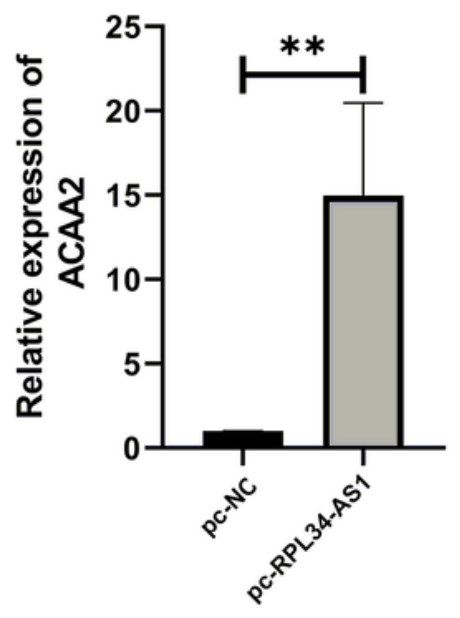

G
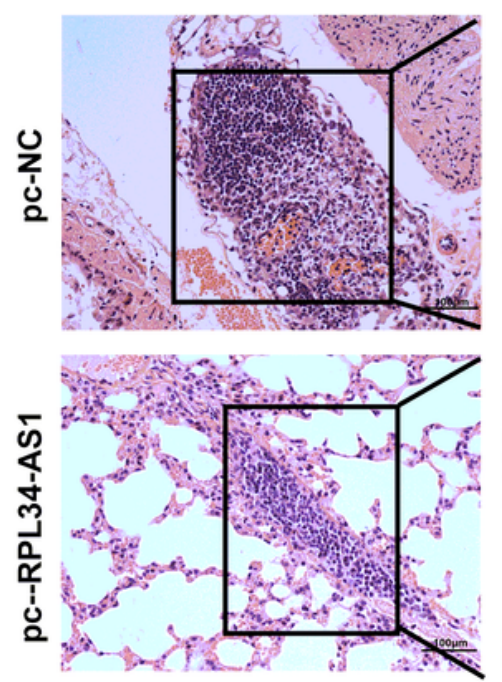

$40 x$
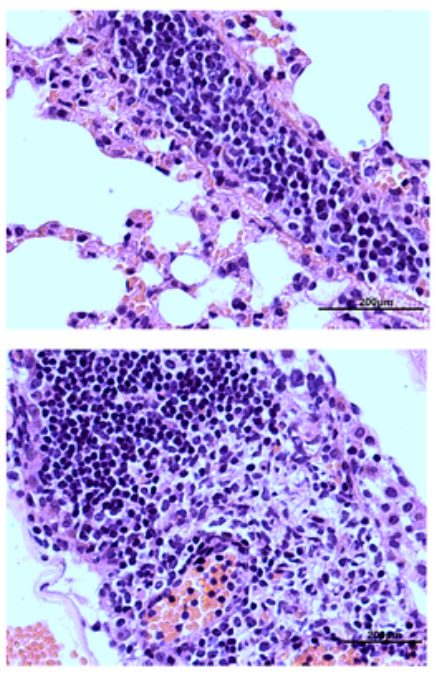

Figure 3 
Overexpression of IncRPL34-AS1 restrains tumorigenesis and growth of ESCC in vivo. a. Image of subcutaneous tumor tissues in IncRPL34-AS1-overexpressing group and control group. b. Analysis of tumor volume of mice measured every three days. c. The relative weights of tumors were evaluated. $d$, e. Relative expression levels of RPL34-AS1 and ACAA2 were observed in subcutaneous tumor tissues by RTqPCR. f. The xenografts were H\&E stained and expression of ki67 was measured by immunohistochemistry. Scale bar, $100 \mu \mathrm{m}$. g. EC109 cells were injected into the tail veins of nude mice. After 34 days, lungs were obtained. Representative lung images of H\&E staining were shown. Scale bar, $100 \mu \mathrm{m}, 200 \mu \mathrm{m}$. Data were showed as mean \pm SD. ${ }^{*} \mathrm{P}<0.05,{ }^{*} \mathrm{P}<0.01$.

A

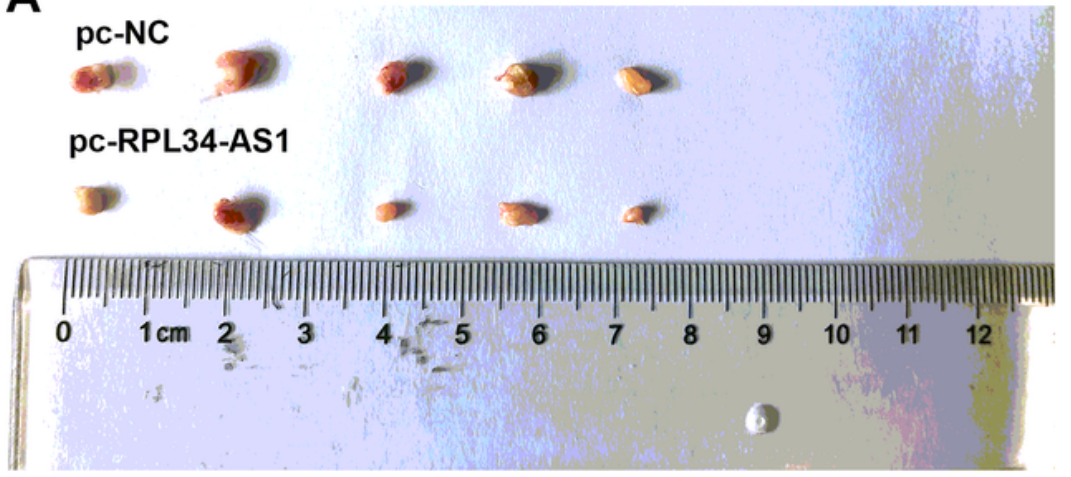

C

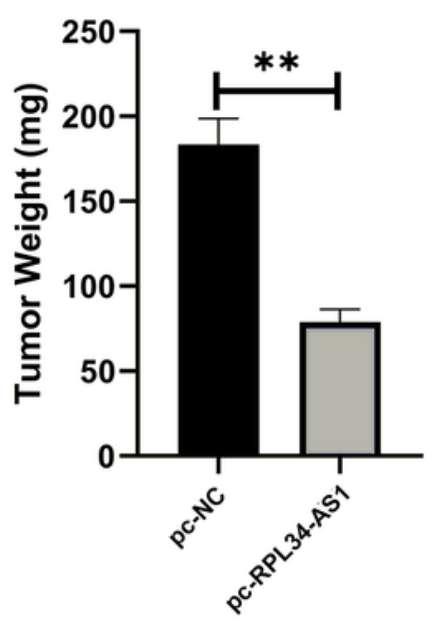

F
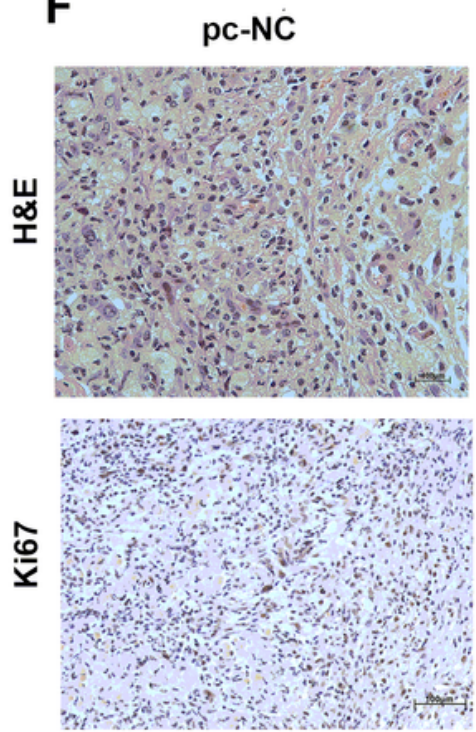

D

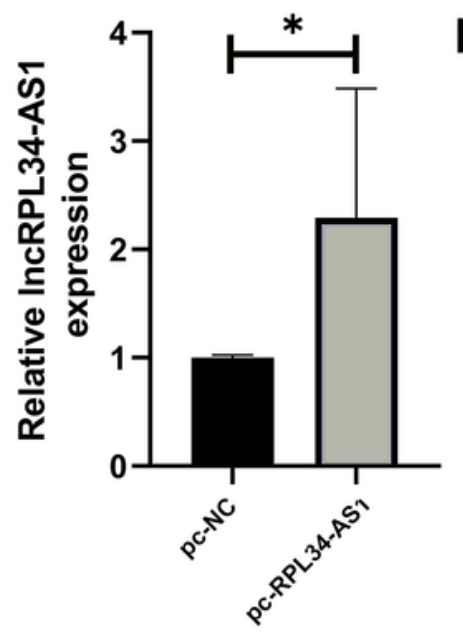

pc-RPL34-AS1
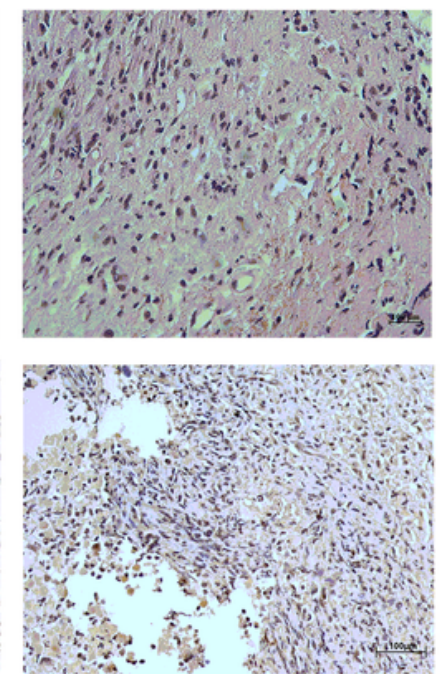

B

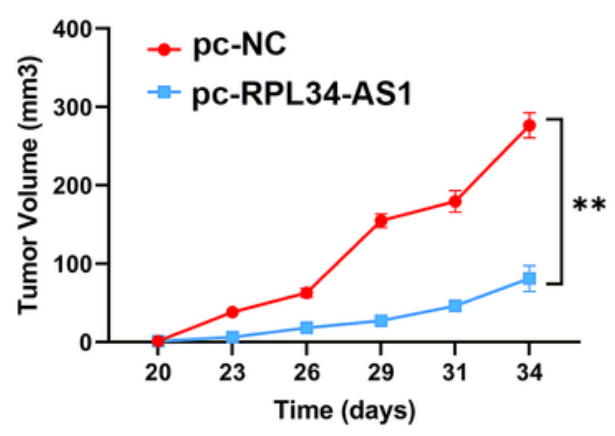

E

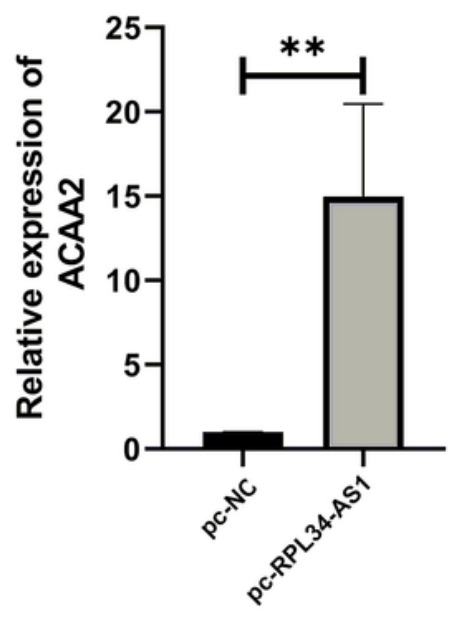

G
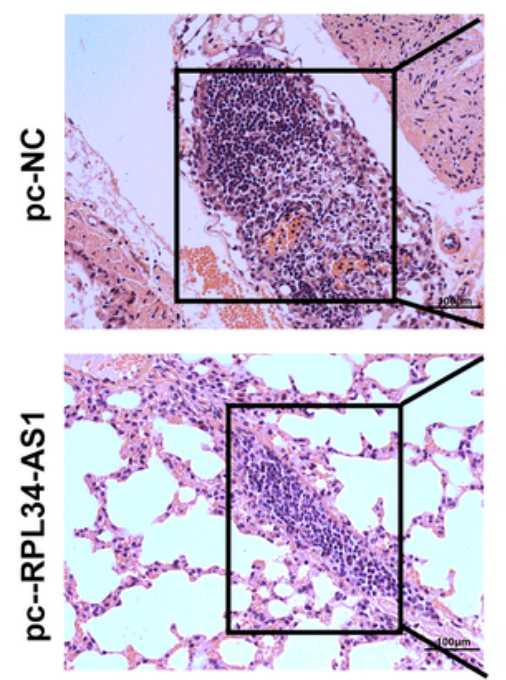

$40 x$
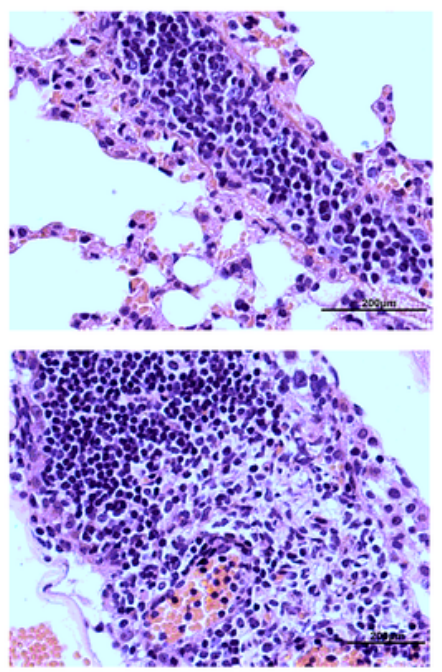


\section{Figure 3}

Overexpression of IncRPL34-AS1 restrains tumorigenesis and growth of ESCC in vivo. a. Image of subcutaneous tumor tissues in IncRPL34-AS1-overexpressing group and control group. b. Analysis of tumor volume of mice measured every three days. c. The relative weights of tumors were evaluated. $d$, e. Relative expression levels of RPL34-AS1 and ACAA2 were observed in subcutaneous tumor tissues by RTqPCR. $f$. The xenografts were H\&E stained and expression of ki67 was measured by immunohistochemistry. Scale bar, $100 \mu \mathrm{m}$. g. EC109 cells were injected into the tail veins of nude mice. After 34 days, lungs were obtained. Representative lung images of H\&E staining were shown. Scale bar, $100 \mu \mathrm{m}, 200 \mu \mathrm{m}$. Data were showed as mean \pm SD. ${ }^{*} \mathrm{P}<0.05,{ }^{*} \mathrm{P}<0.01$. 
A

pc-NC

pc-RPL34-AS1

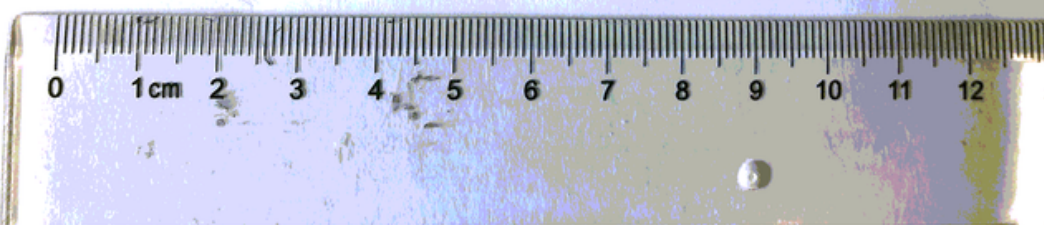

C

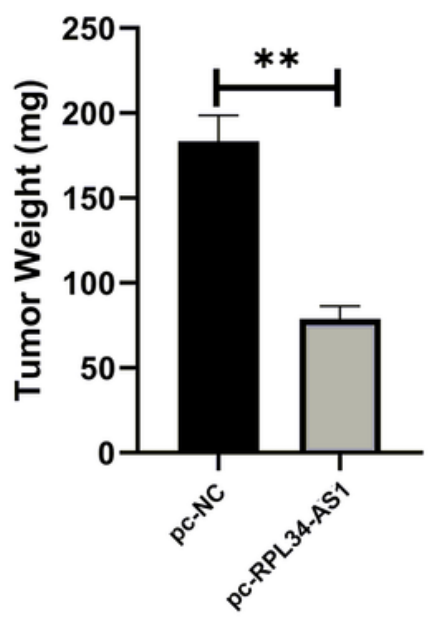

$\mathbf{F}$

pc-NC
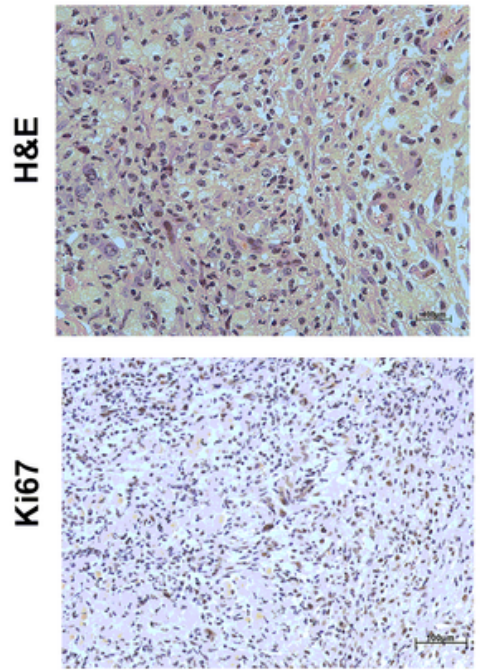

D

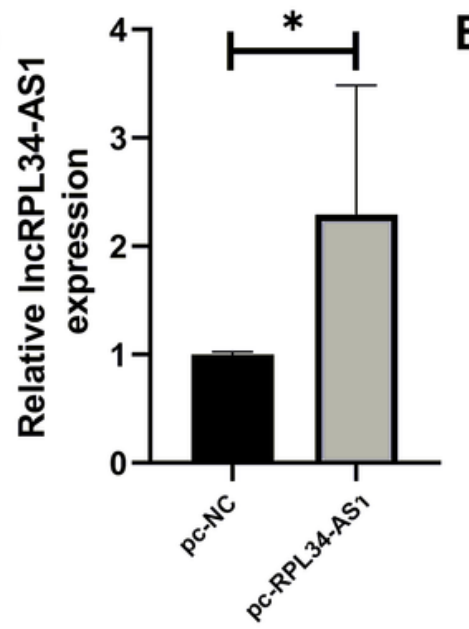

pc-RPL34-AS1
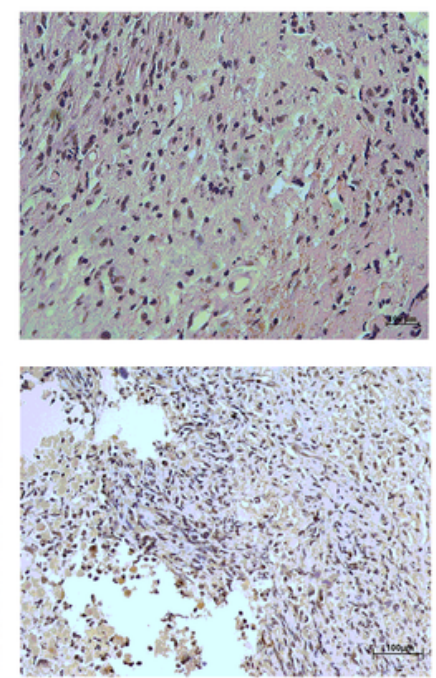

B

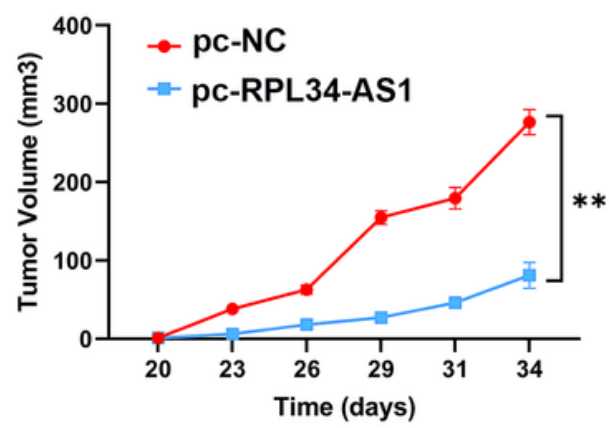

E

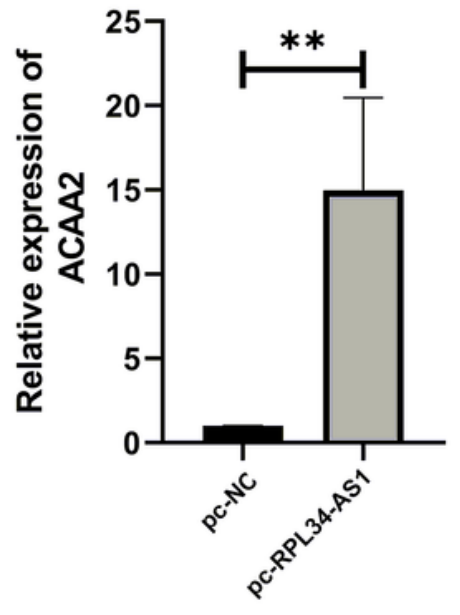

G
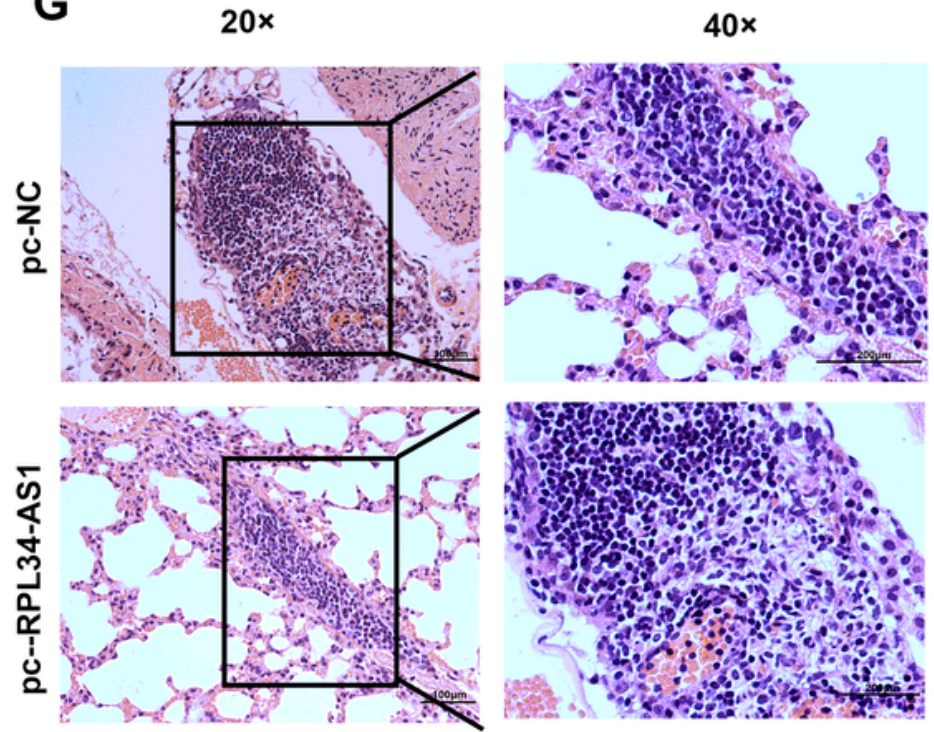

Figure 3

Overexpression of IncRPL34-AS1 restrains tumorigenesis and growth of ESCC in vivo. a. Image of subcutaneous tumor tissues in IncRPL34-AS1-overexpressing group and control group. b. Analysis of tumor volume of mice measured every three days. c. The relative weights of tumors were evaluated. $d$, e. Relative expression levels of RPL34-AS1 and ACAA2 were observed in subcutaneous tumor tissues by RTqPCR. f. The xenografts were H\&E stained and expression of ki67 was measured by 
immunohistochemistry. Scale bar, $100 \mu \mathrm{m}$. g. EC109 cells were injected into the tail veins of nude mice. After 34 days, lungs were obtained. Representative lung images of H\&E staining were shown. Scale bar, $100 \mu \mathrm{m}, 200 \mu \mathrm{m}$. Data were showed as mean \pm SD. ${ }^{*} \mathrm{P}<0.05, * * \mathrm{P}<0.01$.

A

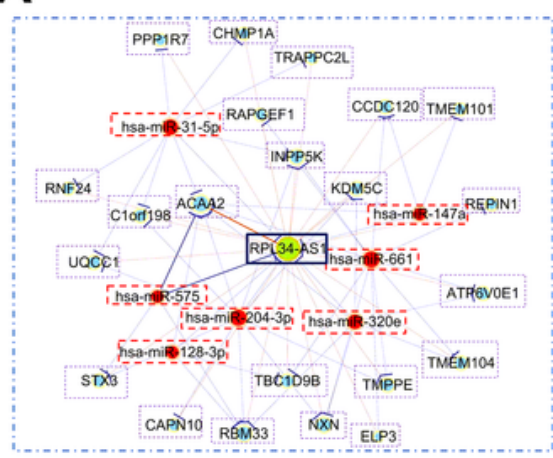

D

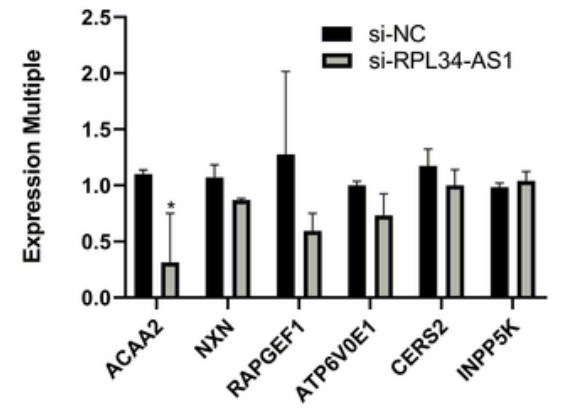

G
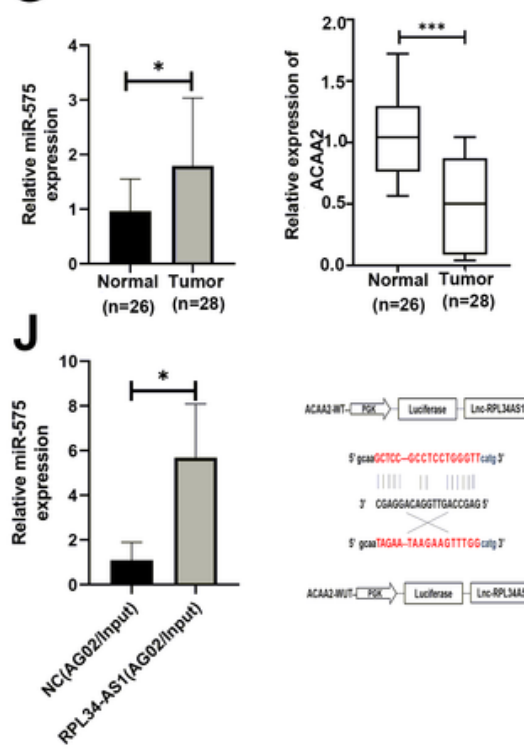

B

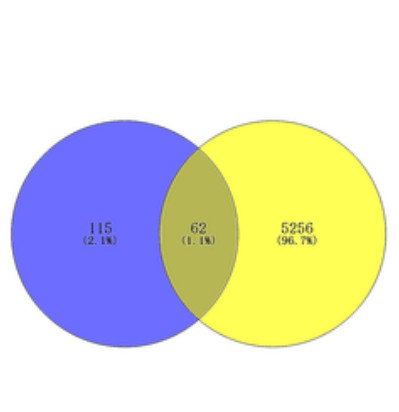

C
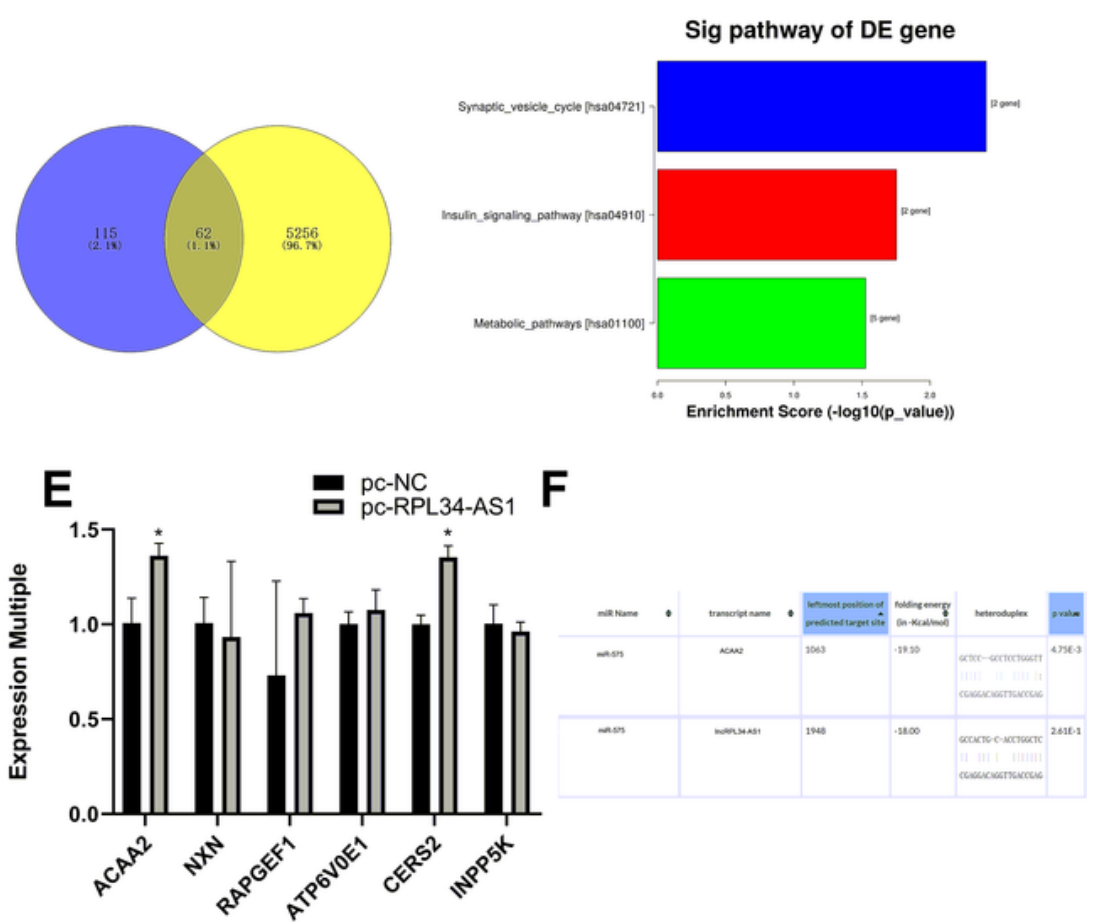

H

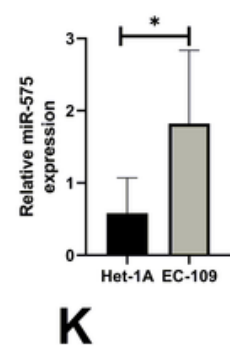

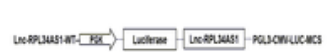
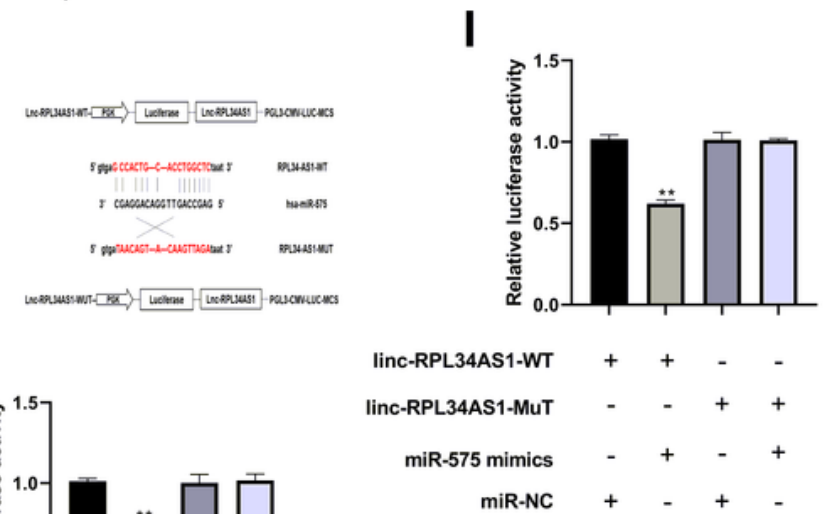

$\mathbf{L}$

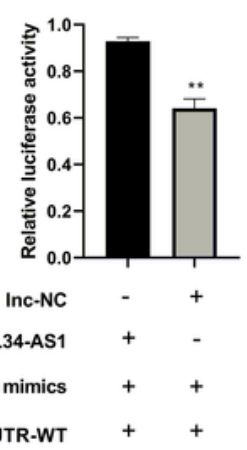

Figure 4

LncRPL34-AS1 serves as a miRNA sponge of miR-575 to regulate ACAA2 expression. a. The IncRPL34AS1-miRNA-mRNA network diagram was screened by miRanda and TargetScan algorithm. b, c. The 
intersection mRNA genes of RNA-seq expressed genes and ceRNA targets were displayed in Venny. The functional pathways were enriched via DAVID bioinformatics resources. $d$, e. The targeted genes expression was detected by RT-qPCR after knockdown and overexpression of IncRPL34-AS1 in EC109 cells. $\mathrm{f}$. The miRbase predicted binding ability between candidate miR-575 and targeting RPL34-AS1 and ACAA2. $\mathrm{g}$, h. The expression of miR- 575 and ACAA2 was performed by RT-qPCR in ESCC tissues and cells. i. Prediction for miR-575 binding elements on IncRPL34-AS1. Mutations were generated in IncRPL34-AS1 binding sites of miR575. Luciferase activity in EC109 cells co-transfected with miR-575 mimics/miR-NC and WT/MuT IncRPL34-AS1. Data were presented as the relative ratio of firefly luciferase activity to Renilla luciferase activity. j. The anti-AGO2 RIP immunoprecipitations were measured by RTqPCR. k. Prediction for miR-575 binding elements on ACAA2. Mutations were generated in ACAA2 3'-UTR binding sites of miR-575. DLR analysis was performed to confirm miR-575 shares binding sites with ACAA2. The luciferase activity was detected in cells co-transfected with miR-575 mimics/miR-NC and wild-type/mutant ACAA2; and (I) to further evaluate the relationship of IncRPL34-AS1, miR575, and ACAA2 by use of DLR analysis. Data were showed as mean $\pm S D$. ${ }^{*} P<0.05, * \star P<0.01, * \star * P<0.001$. 
A
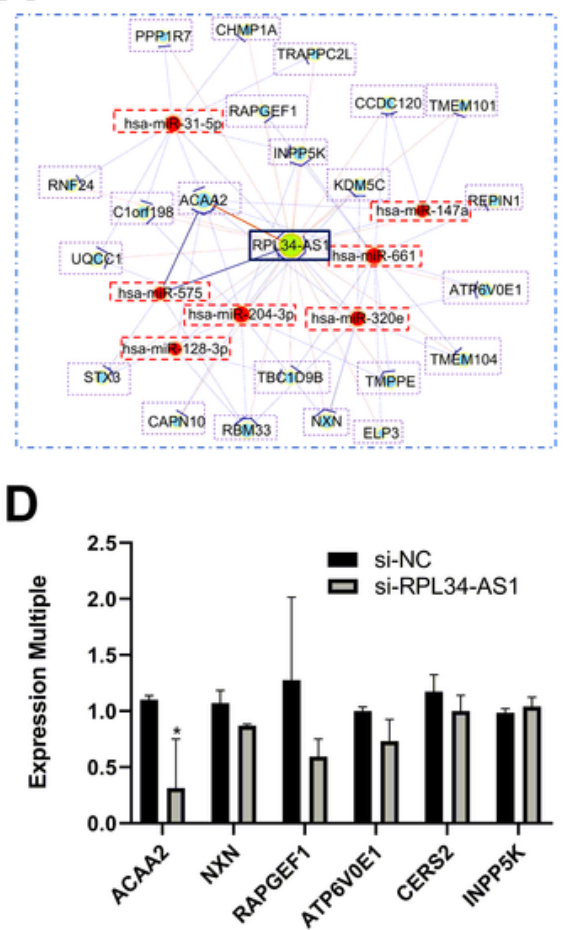

G

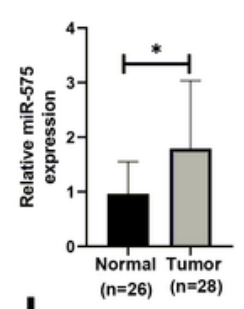

$\mathbf{J}$
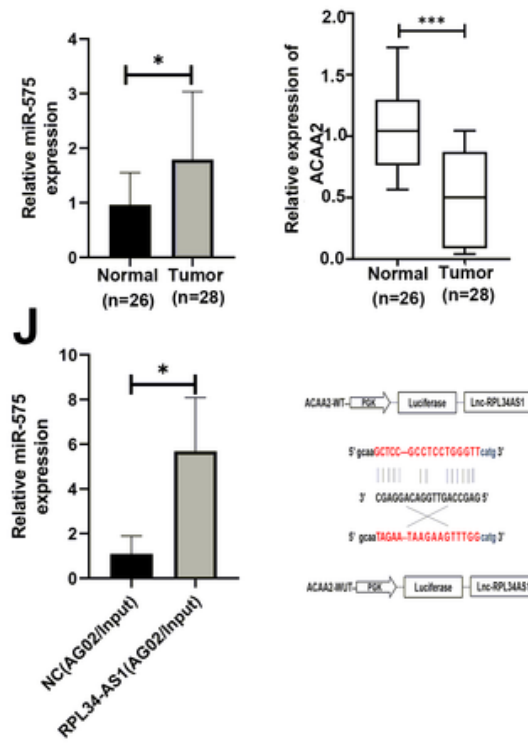

B
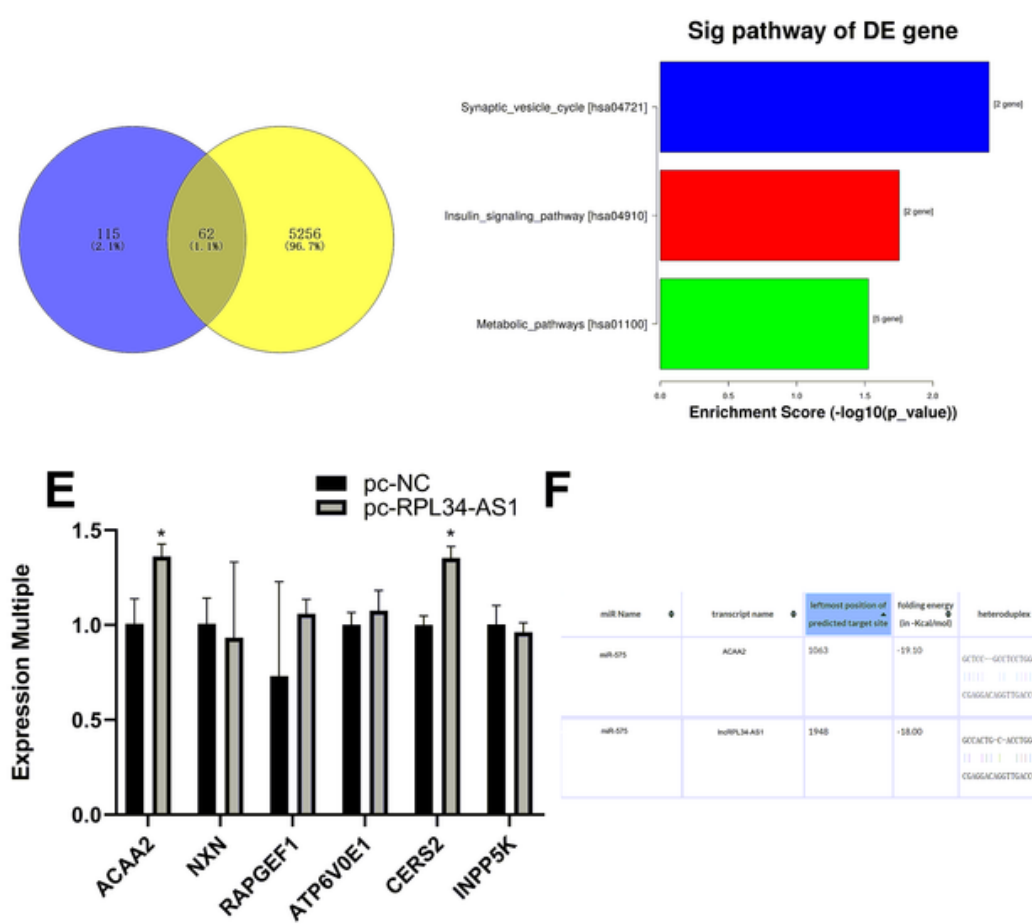

H

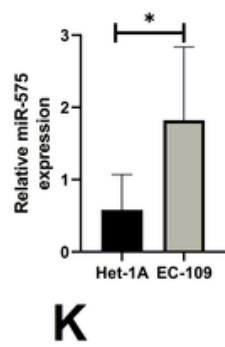

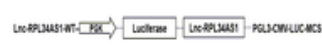
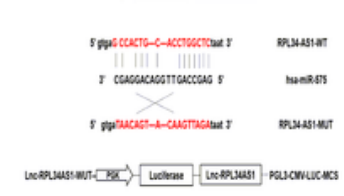

$\mathrm{K}$

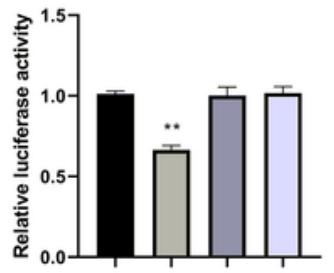

ACAA2 3'UTR-WT

ACAA2 3'UTR-MUT

miR-575 mimics

miR-NC

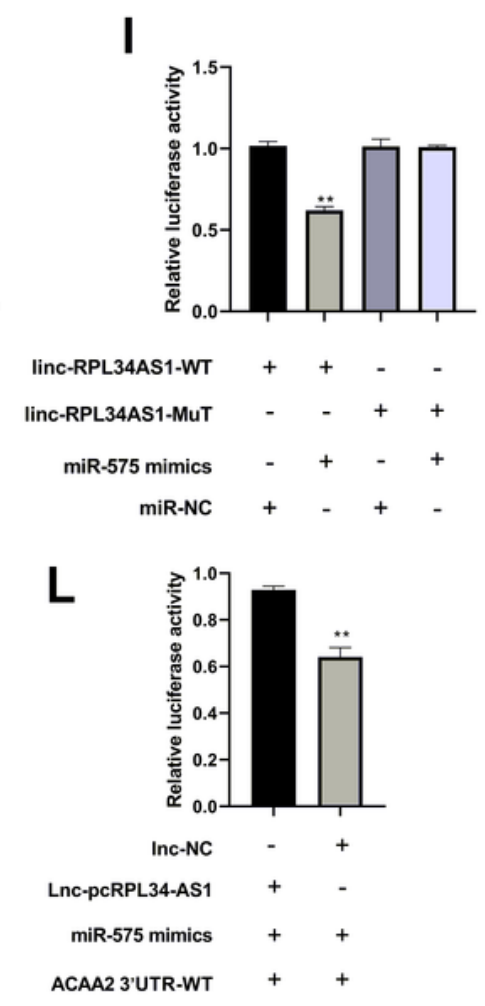

Figure 4

LncRPL34-AS1 serves as a miRNA sponge of miR-575 to regulate ACAA2 expression. a. The IncRPL34AS1-miRNA-mRNA network diagram was screened by miRanda and TargetScan algorithm. b, c. The intersection mRNA genes of RNA-seq expressed genes and ceRNA targets were displayed in Venny. The functional pathways were enriched via DAVID bioinformatics resources. $d$, e. The targeted genes expression was detected by RT-qPCR after knockdown and overexpression of IncRPL34-AS1 in EC109 
cells. $\mathrm{f}$. The miRbase predicted binding ability between candidate miR-575 and targeting RPL34-AS1 and ACAA2. $\mathrm{g}$, $\mathrm{h}$. The expression of miR- 575 and ACAA2 was performed by RT-qPCR in ESCC tissues and cells. i. Prediction for miR-575 binding elements on IncRPL34-AS1. Mutations were generated in IncRPL34-AS1 binding sites of miR575. Luciferase activity in EC109 cells co-transfected with miR-575 mimics/miR-NC and WT/MuT IncRPL34-AS1. Data were presented as the relative ratio of firefly luciferase activity to Renilla luciferase activity. j. The anti-AGO2 RIP immunoprecipitations were measured by RTqPCR. k. Prediction for miR-575 binding elements on ACAA2. Mutations were generated in ACAA2 3'-UTR binding sites of miR-575. DLR analysis was performed to confirm miR- 575 shares binding sites with ACAA2. The luciferase activity was detected in cells co-transfected with miR-575 mimics/miR-NC and wild-type/mutant ACAA2; and (I) to further evaluate the relationship of IncRPL34-AS1, miR575, and ACAA2 by use of DLR analysis. Data were showed as mean $\pm S D$. ${ }^{*} P<0.05,{ }^{\star} P P<0.01$, ${ }^{\star \star \star} P<0.001$. 
A
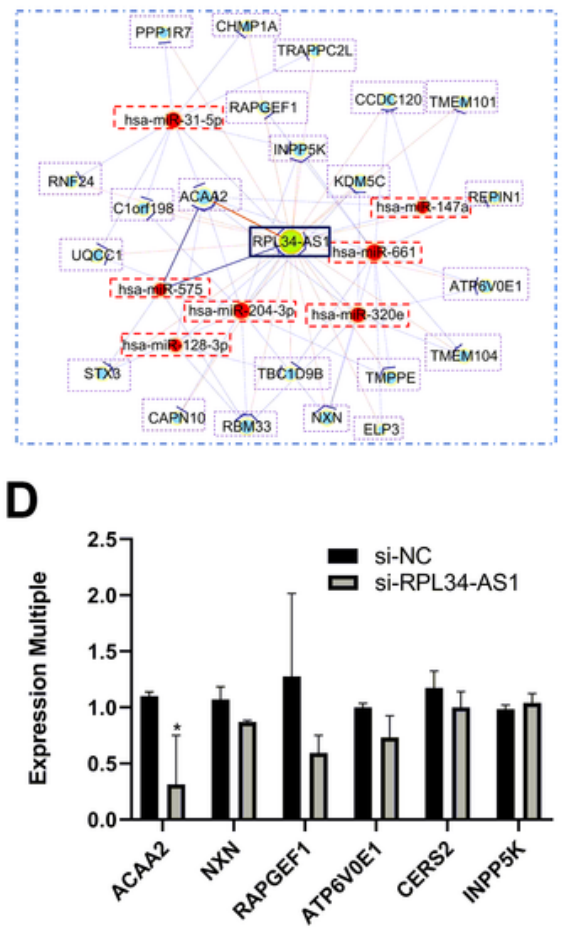

G

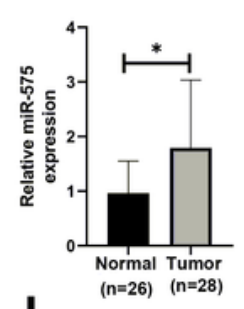

$\mathbf{J}$
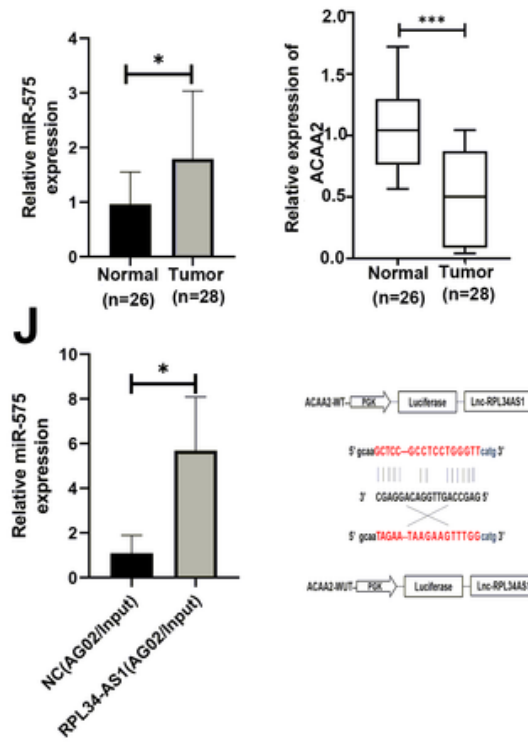

B
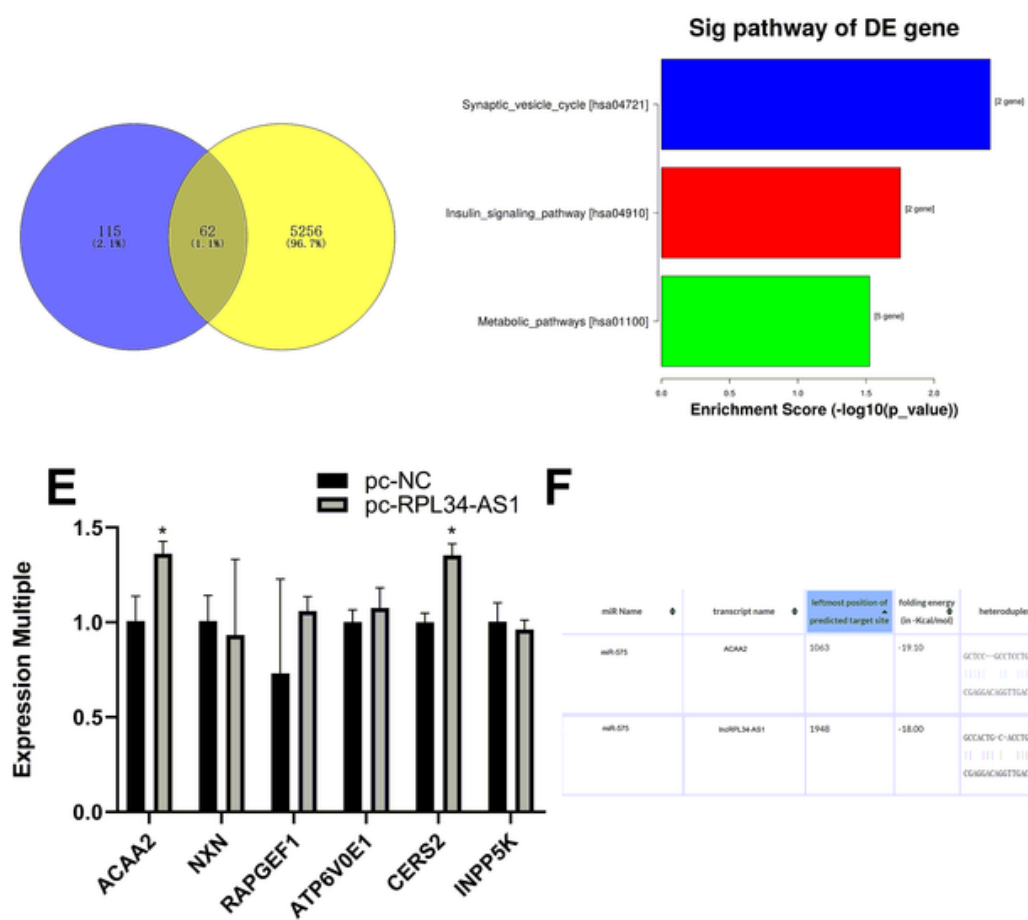

$\mathbf{H}$

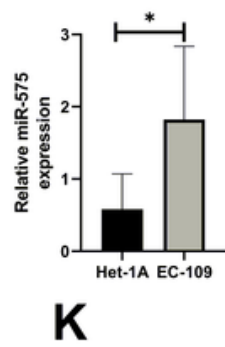

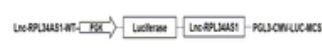
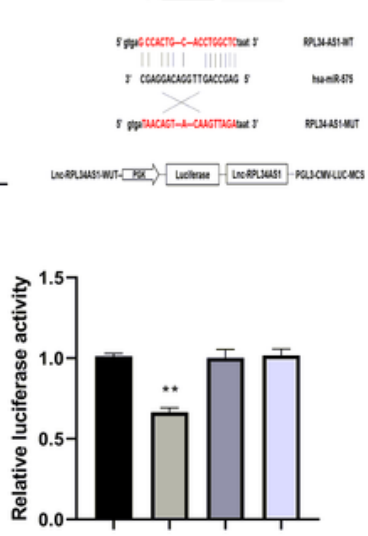

ACAA2 3'UTR-WT

ACAA2 3'UTR-MUT

miR-575 mimic

miR-NC

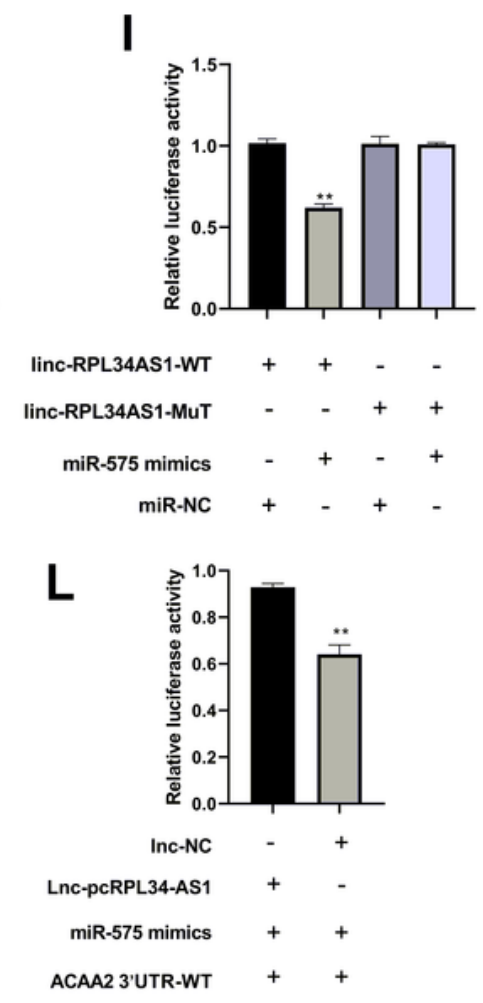

Figure 4

LncRPL34-AS1 serves as a miRNA sponge of miR-575 to regulate ACAA2 expression. a. The IncRPL34AS1-miRNA-mRNA network diagram was screened by miRanda and TargetScan algorithm. b, c. The intersection mRNA genes of RNA-seq expressed genes and ceRNA targets were displayed in Venny. The functional pathways were enriched via DAVID bioinformatics resources. $d$, e. The targeted genes expression was detected by RT-qPCR after knockdown and overexpression of IncRPL34-AS1 in EC109 
cells. $\mathrm{f}$. The miRbase predicted binding ability between candidate miR-575 and targeting RPL34-AS1 and ACAA2. $\mathrm{g}$, $\mathrm{h}$. The expression of miR- 575 and ACAA2 was performed by RT-qPCR in ESCC tissues and cells. i. Prediction for miR-575 binding elements on IncRPL34-AS1. Mutations were generated in IncRPL34-AS1 binding sites of miR575. Luciferase activity in EC109 cells co-transfected with miR-575 mimics/miR-NC and WT/MuT IncRPL34-AS1. Data were presented as the relative ratio of firefly luciferase activity to Renilla luciferase activity. j. The anti-AGO2 RIP immunoprecipitations were measured by RTqPCR. k. Prediction for miR-575 binding elements on ACAA2. Mutations were generated in ACAA2 3'-UTR binding sites of miR-575. DLR analysis was performed to confirm miR- 575 shares binding sites with ACAA2. The luciferase activity was detected in cells co-transfected with miR-575 mimics/miR-NC and wild-type/mutant ACAA2; and (I) to further evaluate the relationship of IncRPL34-AS1, miR575, and ACAA2 by use of DLR analysis. Data were showed as mean $\pm S D$. ${ }^{*} P<0.05,{ }^{\star} P P<0.01$, ${ }^{\star \star \star} P<0.001$. 


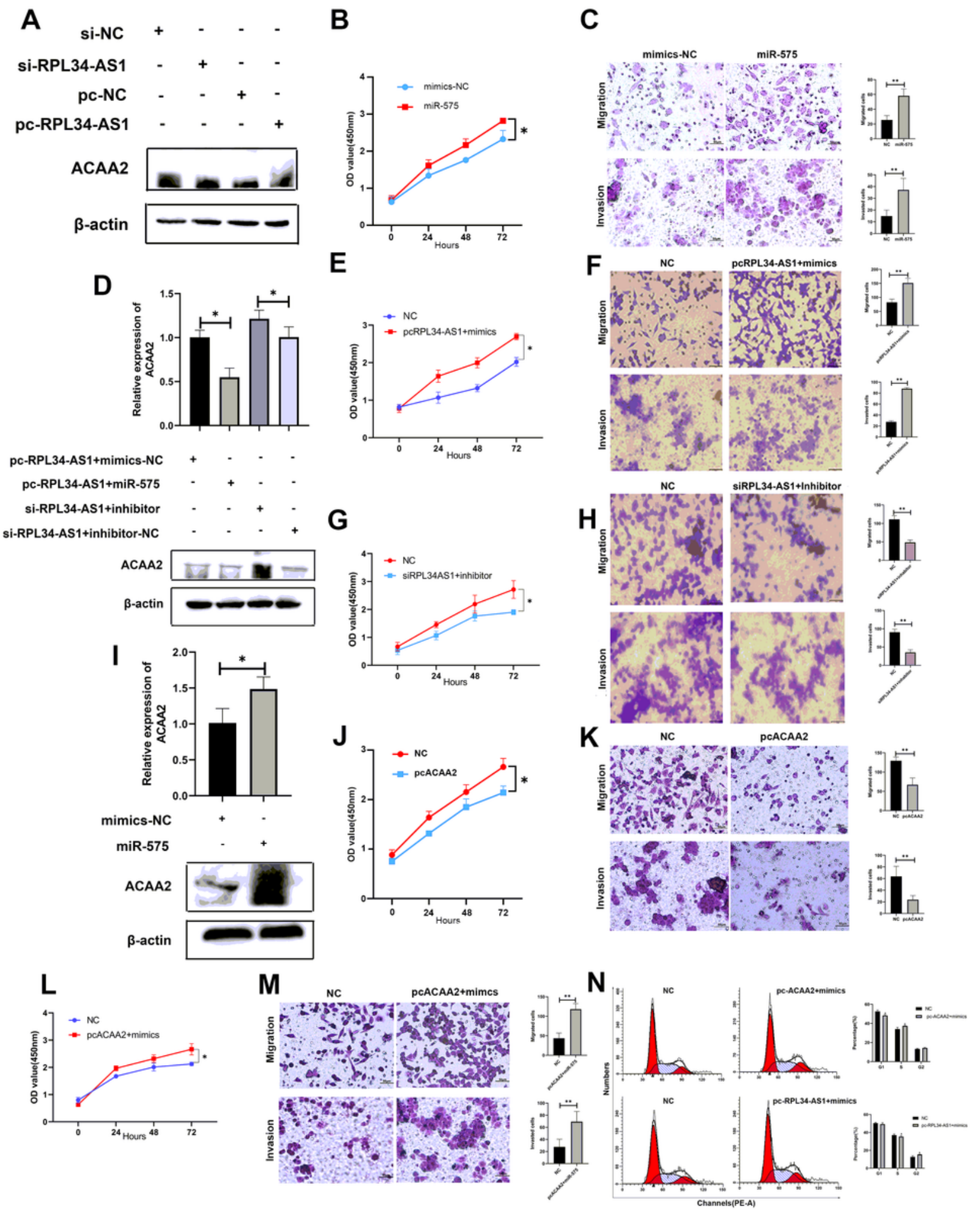

Figure 5

LncRPL34-AS1 suppresses ESCC cell growth and metastasis through IncRPL34-AS1/miR-575/ACAA2 axis. a. Relative protein levels of ACAA2 in EC109 cells transfected with si-NC, si-Inc, pc-NC and pc-Inc by western blot assay. b, c. CCK-8 assays were performed to determine the ability of proliferation and cell migratory and invasive capabilities were assessed by transwell assays in EC109 cells transfected with miR-575 and mimics-NC. Scale bar, $50 \mu \mathrm{m}$. d. Relative mRNA and protein levels of ACAA2 were evaluated 
by RT-qPCR and western blot in EC109 cells transfected with indicated mimics, inhibitor, NC, si-Inc or pcInc, respectively. e-h. CCK-8 assays were performed to determine the ability of proliferation and cell migratory and invasive capabilities were assessed by transwell assays in EC109 cells transfected with indicated mimics, inhibitor, NC, si-Inc or pc-Inc, respectively. Scale bar, $50 \mu \mathrm{m}$. I. Relative mRNA and protein levels of ACAA2 were evaluated by RT-qPCR and western blot in EC109 cells transfected with the miR-575 mimics. j-m. CCK-8 assays were performed to determine the ability of proliferation and cell migratory and invasive capabilities were assessed by transwell assays in EC109 cells transfected with mimics, mimics NC, pcACAA2 and pc-NC. n. Flow cytometry was performed to determine the effect cotransfected by mimics, mi-NC, pc-Inc or pcACAA2 on cell cycle by flow cytometry analysis. Data were showed as mean $\pm \mathrm{SD}$. ${ }^{*} \mathrm{P}<0.05,{ }^{* *} \mathrm{P}<0.01$. 


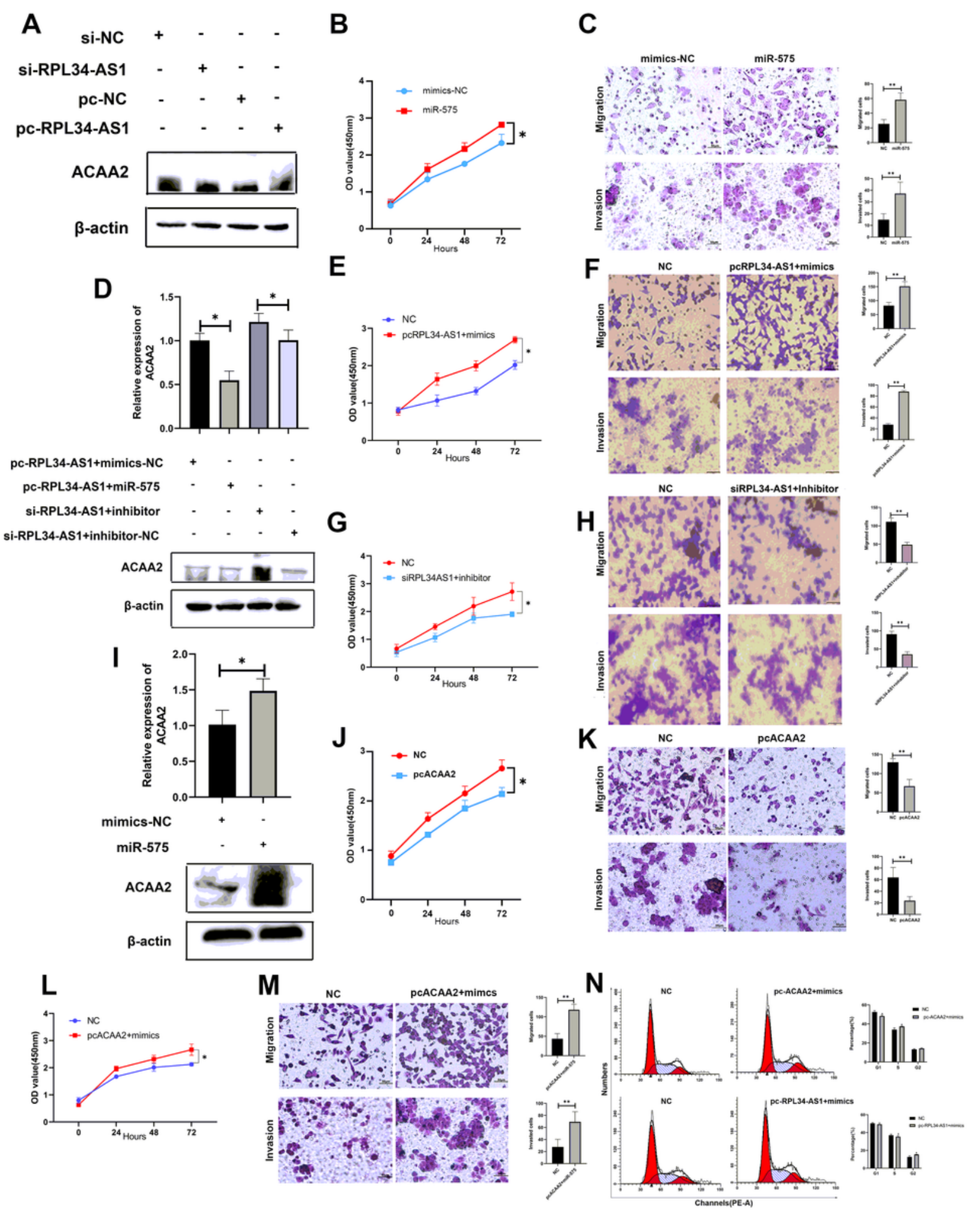

Figure 5

LncRPL34-AS1 suppresses ESCC cell growth and metastasis through IncRPL34-AS1/miR-575/ACAA2 axis. a. Relative protein levels of ACAA2 in EC109 cells transfected with si-NC, si-Inc, pc-NC and pc-Inc by western blot assay. b, c. CCK-8 assays were performed to determine the ability of proliferation and cell migratory and invasive capabilities were assessed by transwell assays in EC109 cells transfected with miR-575 and mimics-NC. Scale bar, $50 \mu \mathrm{m}$. d. Relative mRNA and protein levels of ACAA2 were evaluated 
by RT-qPCR and western blot in EC109 cells transfected with indicated mimics, inhibitor, NC, si-Inc or pcInc, respectively. e-h. CCK-8 assays were performed to determine the ability of proliferation and cell migratory and invasive capabilities were assessed by transwell assays in EC109 cells transfected with indicated mimics, inhibitor, NC, si-Inc or pc-Inc, respectively. Scale bar, $50 \mu \mathrm{m}$. I. Relative mRNA and protein levels of ACAA2 were evaluated by RT-qPCR and western blot in EC109 cells transfected with the miR-575 mimics. j-m. CCK-8 assays were performed to determine the ability of proliferation and cell migratory and invasive capabilities were assessed by transwell assays in EC109 cells transfected with mimics, mimics NC, pcACAA2 and pc-NC. n. Flow cytometry was performed to determine the effect cotransfected by mimics, mi-NC, pc-Inc or pcACAA2 on cell cycle by flow cytometry analysis. Data were showed as mean $\pm \mathrm{SD}$. ${ }^{*} \mathrm{P}<0.05,{ }^{* *} \mathrm{P}<0.01$. 


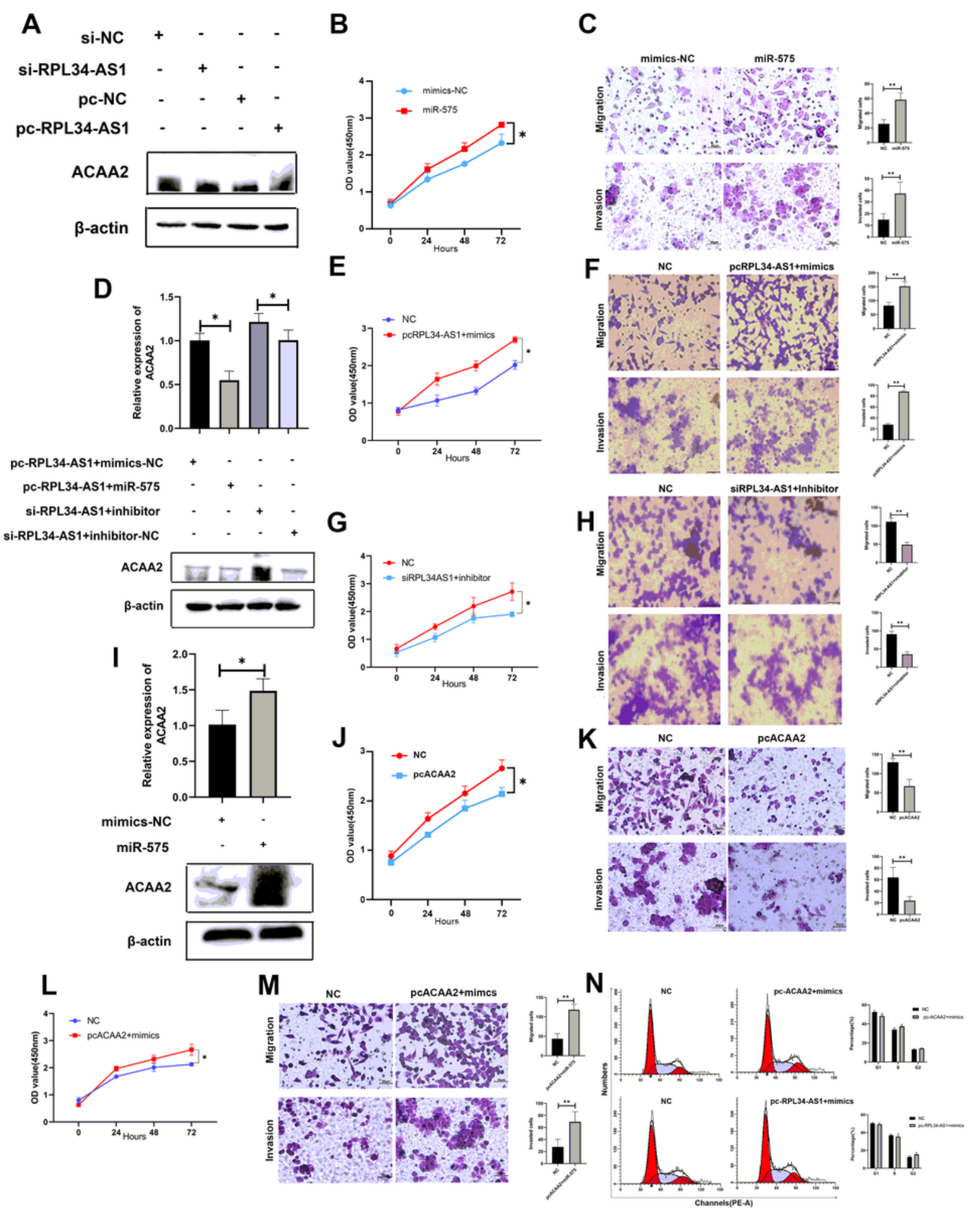

Figure 5

LncRPL34-AS1 suppresses ESCC cell growth and metastasis through IncRPL34-AS1/miR-575/ACAA2 axis. a. Relative protein levels of ACAA2 in EC109 cells transfected with si-NC, si-Inc, pc-NC and pc-Inc by western blot assay. b, c. CCK-8 assays were performed to determine the ability of proliferation and cell migratory and invasive capabilities were assessed by transwell assays in EC109 cells transfected with miR-575 and mimics-NC. Scale bar, $50 \mu \mathrm{m}$. d. Relative mRNA and protein levels of ACAA2 were evaluated 
by RT-qPCR and western blot in EC109 cells transfected with indicated mimics, inhibitor, NC, si-Inc or pcInc, respectively. e-h. CCK-8 assays were performed to determine the ability of proliferation and cell migratory and invasive capabilities were assessed by transwell assays in EC109 cells transfected with indicated mimics, inhibitor, NC, si-Inc or pc-Inc, respectively. Scale bar, $50 \mu \mathrm{m}$. I. Relative mRNA and protein levels of ACAA2 were evaluated by RT-qPCR and western blot in EC109 cells transfected with the miR-575 mimics. j-m. CCK-8 assays were performed to determine the ability of proliferation and cell migratory and invasive capabilities were assessed by transwell assays in EC109 cells transfected with mimics, mimics NC, pcACAA2 and pc-NC. n. Flow cytometry was performed to determine the effect cotransfected by mimics, mi-NC, pc-Inc or pcACAA2 on cell cycle by flow cytometry analysis. Data were showed as mean $\pm \mathrm{SD}$. ${ }^{*} \mathrm{P}<0.05,{ }^{* *} \mathrm{P}<0.01$. 


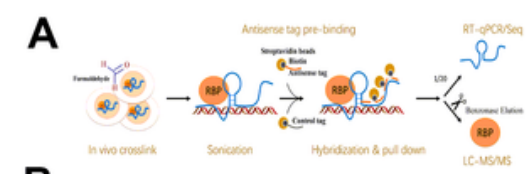

B

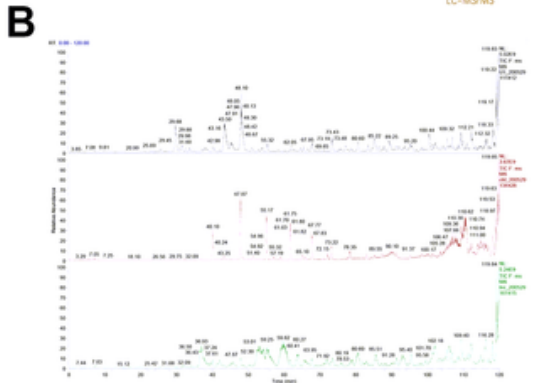

C

$\mathrm{E}$
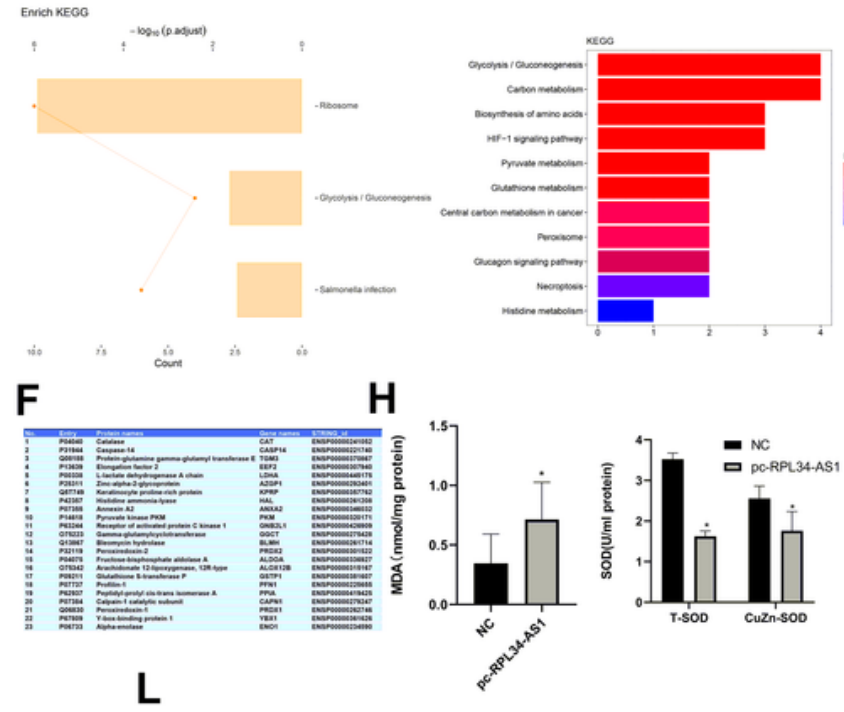

$\mathbf{L}$
D

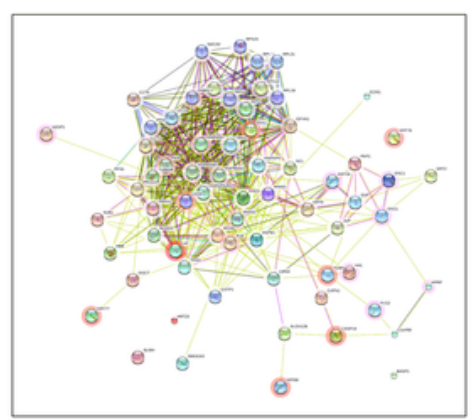

I

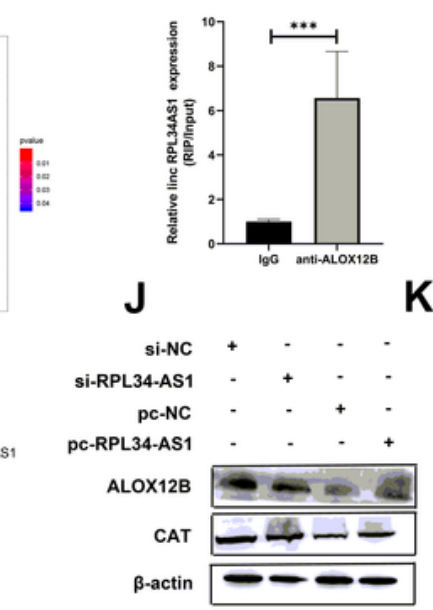

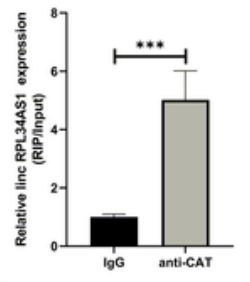

K

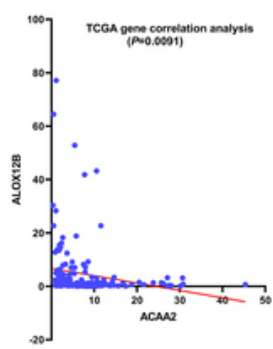

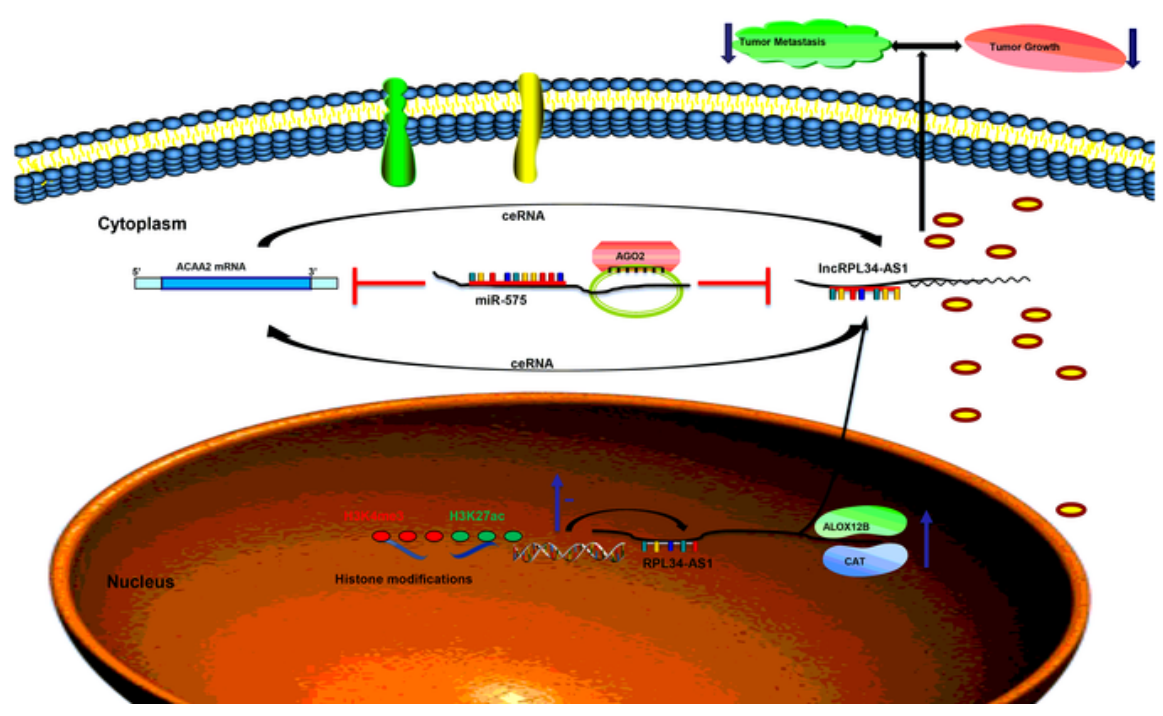

\section{Figure 6}

LncRPL34-AS1 affects biological processes via binding to protein ALOX12B and CAT. a. ChIRP-MS diagram. b. The total TIC peaks of Positive Control (U1 snRNA), Negative control (Ctrl) and Test sample (Lnc). c. Protein enrichment summary with Venn diagram. d. STRINGdb protein-protein network enrichment analysis for all mapped proteins. e. The interaction binding protein enrichment pathways were analyzed by KEGG pathway. f, g. The significantly functional proteins related to RPL34-AS1 and 
enrichment KEGG pathways. h. The oxidative stress damage was detected by MDA and SOD after transfected with overexpression of IncRPL34-AS1. i. RIP experiments for ALOX12B and CAT were performed and the coprecipitated RNA was subjected to RT-qPCR for RPL34-AS1. The fold enrichment of RPL34-AS1 in RIPs was relative to its matching IgG control RIP. j. Western blot was used to investigate the relationship between IncRPL34-AS1 and ALOX12B or CAT at protein levels. $k$. The mRNA correlation between ALOX12B and ACAA2 was confirmed in ESCC of TCGA database. I. A proposed model for illustrating the function and mechanism of RPL34-AS1 in ESCC growth and metastasis. Data were showed as mean \pm SD. ${ }^{*} P<0.05, * * P<0.01$. 


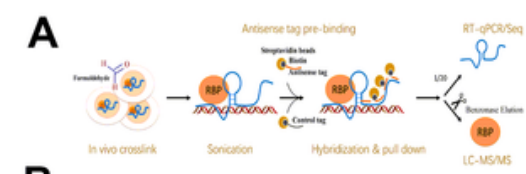

B

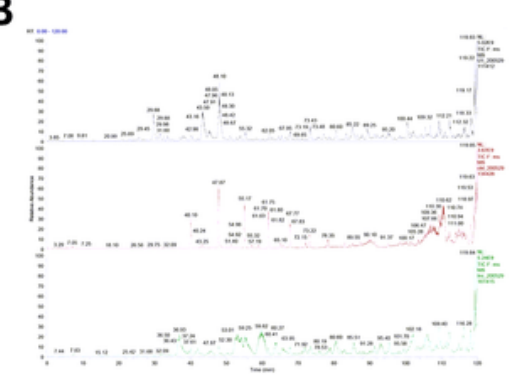

E
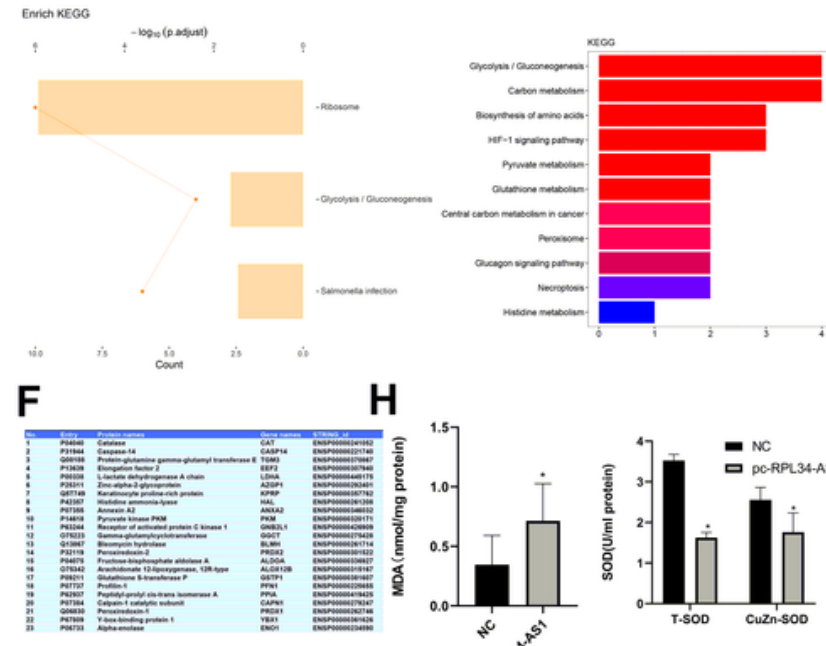

L

政
C

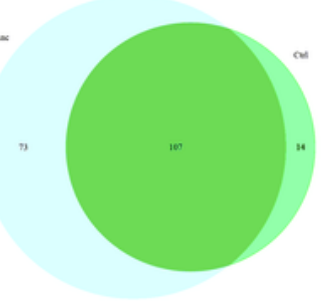

D

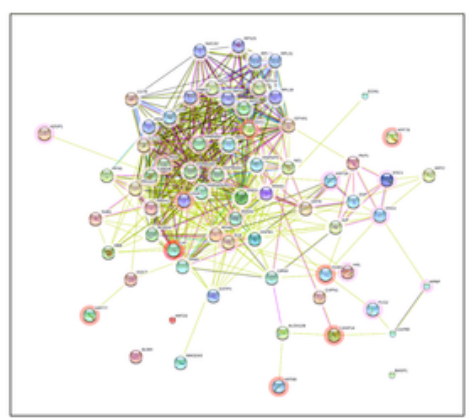

I

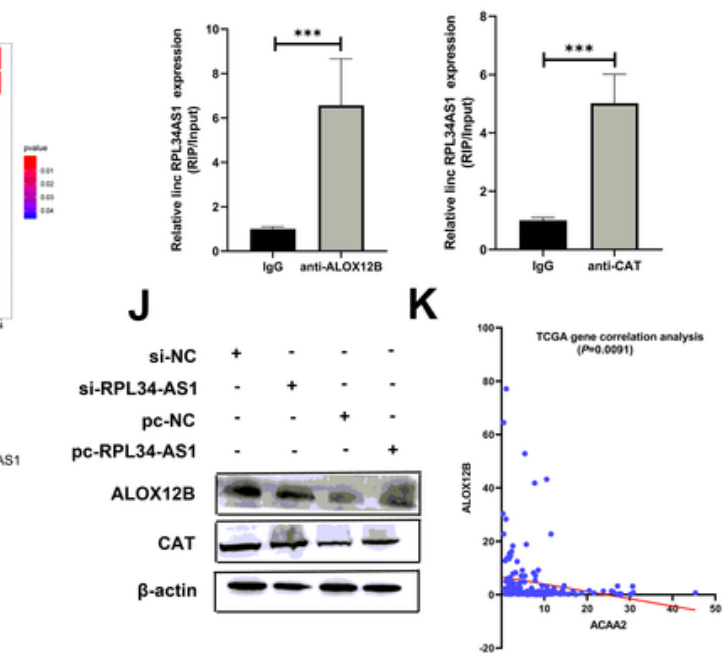

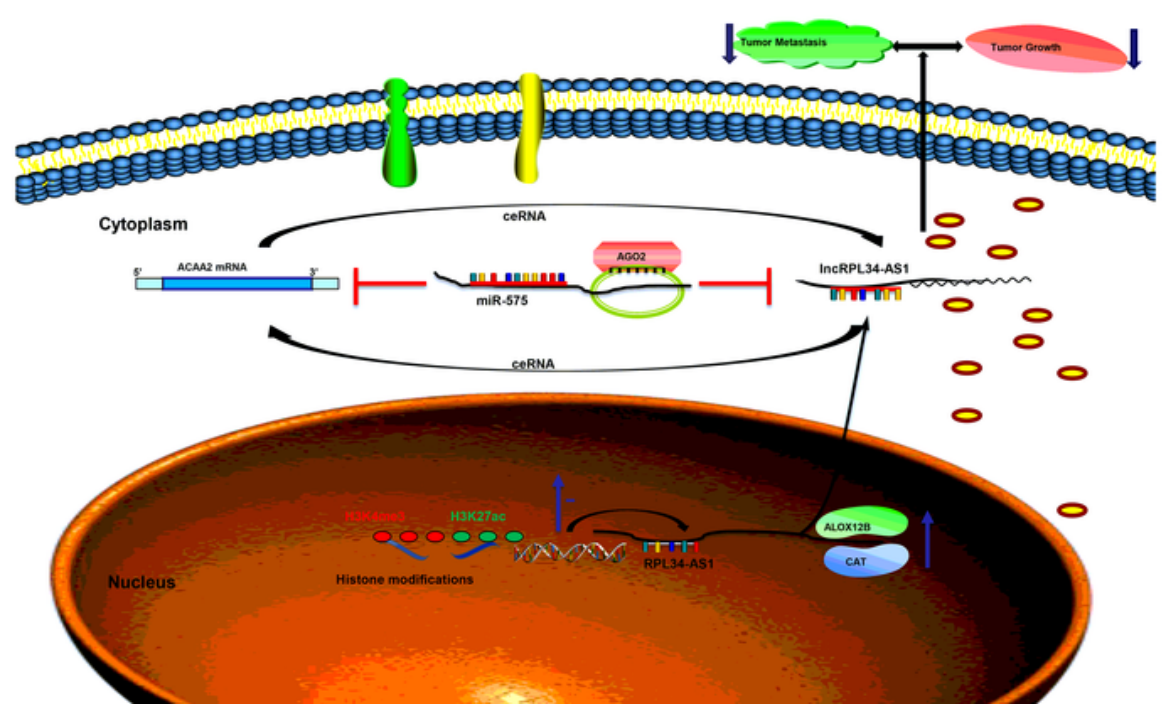

\section{Figure 6}

LncRPL34-AS1 affects biological processes via binding to protein ALOX12B and CAT. a. ChIRP-MS diagram. b. The total TIC peaks of Positive Control (U1 snRNA), Negative control (Ctrl) and Test sample (Lnc). c. Protein enrichment summary with Venn diagram. d. STRINGdb protein-protein network enrichment analysis for all mapped proteins. e. The interaction binding protein enrichment pathways were analyzed by KEGG pathway. f, g. The significantly functional proteins related to RPL34-AS1 and 
enrichment KEGG pathways. h. The oxidative stress damage was detected by MDA and SOD after transfected with overexpression of IncRPL34-AS1. i. RIP experiments for ALOX12B and CAT were performed and the coprecipitated RNA was subjected to RT-qPCR for RPL34-AS1. The fold enrichment of RPL34-AS1 in RIPs was relative to its matching IgG control RIP. j. Western blot was used to investigate the relationship between IncRPL34-AS1 and ALOX12B or CAT at protein levels. $k$. The mRNA correlation between ALOX12B and ACAA2 was confirmed in ESCC of TCGA database. I. A proposed model for illustrating the function and mechanism of RPL34-AS1 in ESCC growth and metastasis. Data were showed as mean \pm SD. ${ }^{*} P<0.05, * * P<0.01$. 


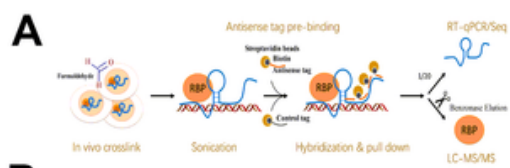

B

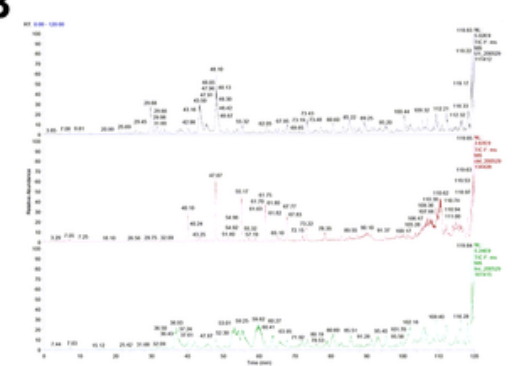

$\mathrm{E}$
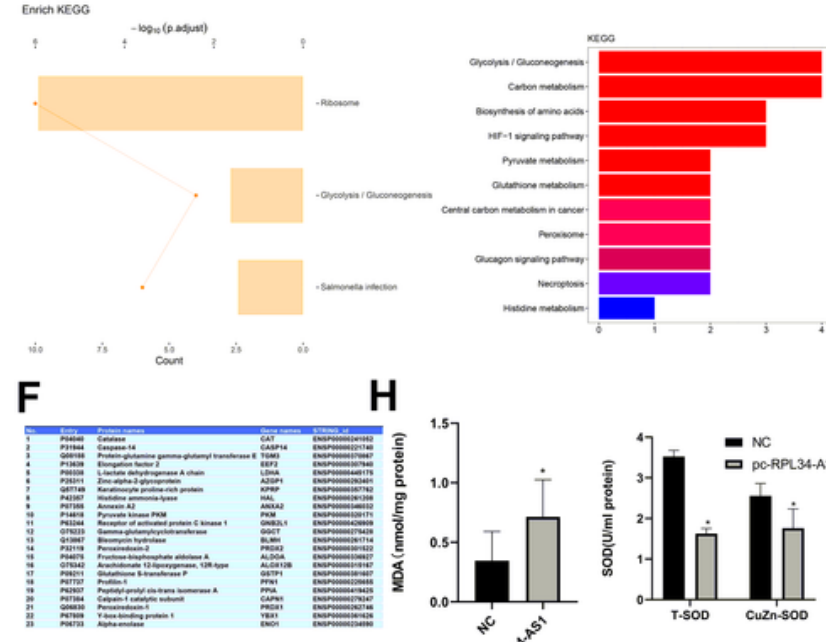

$\mathbf{L}$
C

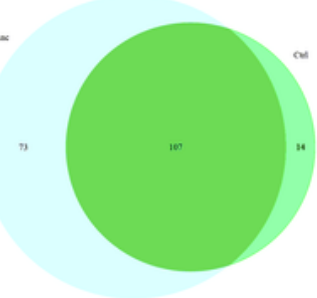

D

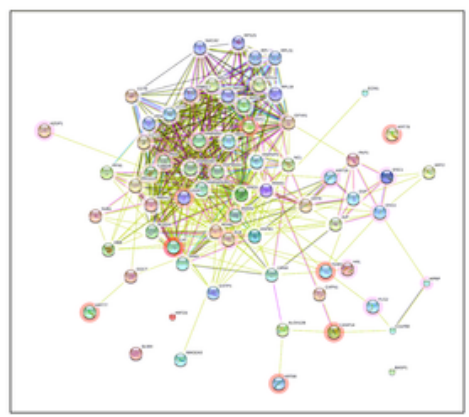

I
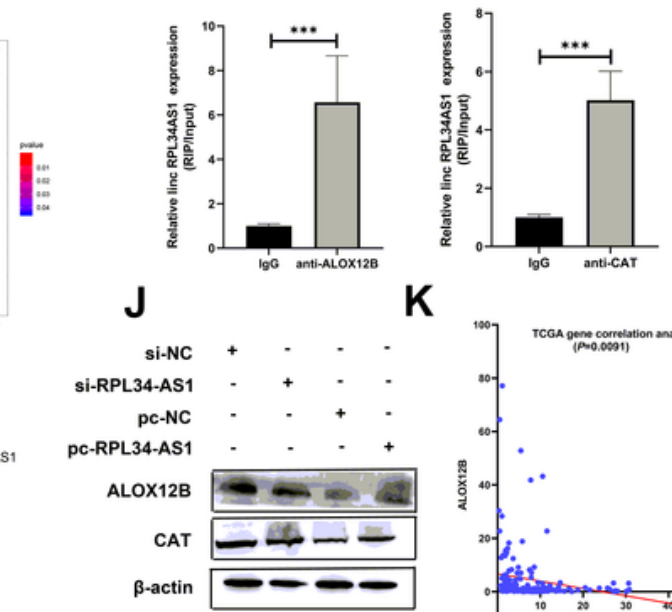

K

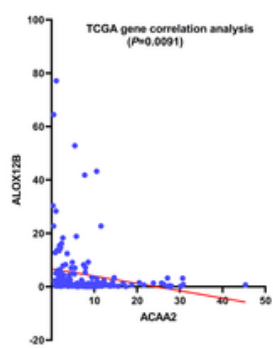

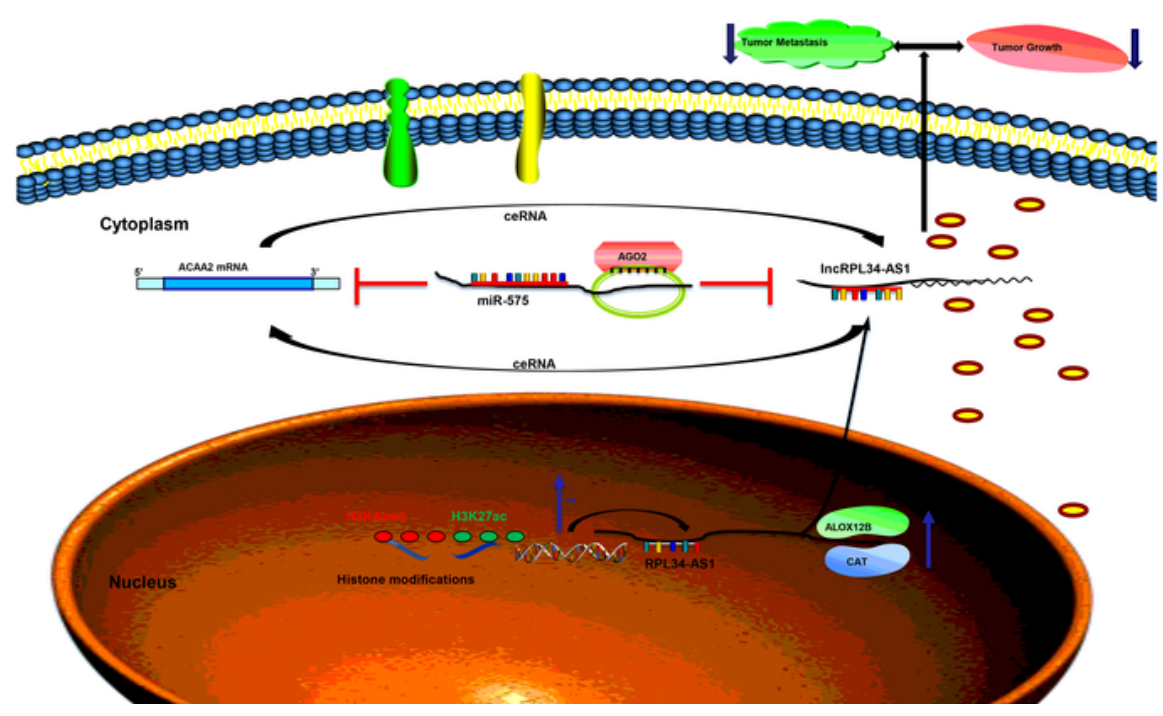

\section{Figure 6}

LncRPL34-AS1 affects biological processes via binding to protein ALOX12B and CAT. a. ChIRP-MS diagram. b. The total TIC peaks of Positive Control (U1 snRNA), Negative control (Ctrl) and Test sample (Lnc). c. Protein enrichment summary with Venn diagram. d. STRINGdb protein-protein network enrichment analysis for all mapped proteins. e. The interaction binding protein enrichment pathways were analyzed by KEGG pathway. f, g. The significantly functional proteins related to RPL34-AS1 and 
enrichment KEGG pathways. h. The oxidative stress damage was detected by MDA and SOD after transfected with overexpression of IncRPL34-AS1. i. RIP experiments for ALOX12B and CAT were performed and the coprecipitated RNA was subjected to RT-qPCR for RPL34-AS1. The fold enrichment of RPL34-AS1 in RIPs was relative to its matching IgG control RIP. j. Western blot was used to investigate the relationship between IncRPL34-AS1 and ALOX12B or CAT at protein levels. $k$. The mRNA correlation between ALOX12B and ACAA2 was confirmed in ESCC of TCGA database. I. A proposed model for illustrating the function and mechanism of RPL34-AS1 in ESCC growth and metastasis. Data were showed as mean \pm SD. ${ }^{*} P<0.05, * * P<0.01$.

\section{Supplementary Files}

This is a list of supplementary files associated with this preprint. Click to download.

- Supplementarylnformation.pdf

- Supplementarylnformation.pdf

- Supplementarylnformation.pdf 